\title{
Sur les $K$-nombres de Pisot de petite mesure
}

\author{
par \\ TOufik ZAÏMI (Riyadh)
}

Introduction. Soient $K$ un corps de nombres et $\theta$ un entier algébrique de module $>1$ et de polynôme minimal $P$ sur $K$. Alors $\theta$ est dit $K$-nombre de Pisot (resp. de Salem) si pour tout plongement $\sigma$ de $K$ dans $\mathbb{C}$ le polynôme $\sigma P$ possède une unique racine de module $>1$ et aucune (resp. au moins une) racine de module 1 . Ces nombres ont été définis par A. M. Bergé et J. Martinet [1] et ont été étudiés par M. J. Bertin qui a considéré le cas où $K$ est un corps quadratique réel [2].

On considère ici deux cas : où $K$ est un corps quadratique imaginaire et où $K$ est un corps cubique totalement réel. Le but est de déterminer des polynômes réciproques de petite mesure.

On commence par déterminer les $K$-nombres de Pisot de petite mesure dans ces deux cas. Ces résultats sont présentés dans le théorème 2 , où on détermine tous les $K$-nombres de Pisot non réels de mesure $<2$ dans le cas où $K$ est quadratique imaginaire, et dans le théorème 4 , où on détermine tous les $K$-nombres de Pisot qui ne sont pas des $\mathbb{Q}$-nombres de Pisot, de mesure $<4$, où $K$ est un corps cubique totalement réel.

La preuve du théorème 2 est basée sur les transformées de Schur et celle du théorème 4 sur l'algorithme de Schur généralisé [2]. Ensuite, en utilisant la construction de Salem, on détermine des $K$-nombres de Salem de petite mesure pour ces deux cas. Dans le cas quadratique imaginaire en appliquant la construction de Salem aux $K$-nombres de Pisot du théorème 2 on trouve des $K$-nombres de Salem non réels de mesure $<1.69$, et dans le cas cubique totalement réel en appliquant cette construction aux $K$-nombres de Pisot du théorème 4 on trouve un seul $K$-nombre de Salem de mesure $<3$, les autres polynômes ont 1 ou 2 conjugués hors du disque unité.

Les calculs sont faits grâce au système Pari [9].

\section{Rappels et résultats préliminaires}

DÉfinition 1. Soit $\theta$ un entier algébrique de polynôme minimal $F$ sur $\mathbb{Q}$ tel que $F(z)=\left(z-\theta_{1}\right)\left(z-\theta_{2}\right) \ldots\left(z-\theta_{n}\right)$. La mesure de $\theta$ (ou bien 
de $F$ ) est le nombre réel $M(\theta)$ (ou bien $M(F)$ ) défini par

$$
M(\theta)=\prod_{1 \leq i \leq n} \max \left\{1,\left|\theta_{i}\right|\right\} .
$$

DÉfinition 2 [1]. Soit $K$ un corps de nombres. Un entier algébrique $\theta$ de module $>1$ est dit $K$-nombre de Pisot (resp. de Salem) si au dessus de tout plongement de $K$ dans $\mathbb{C}$ il admet un unique conjugué de module $>1$ et (resp. au moins un) aucun conjugué de module 1.

La valeur absolue d'un $\mathbb{Q}$-nombre de Pisot (resp. de Salem) est un nombre de Pisot (resp. de Salem).

Généralisation de la construction de Salem. Dans tout ce qui suit, $K$ désigne un corps quadratique ou bien un corps de nombres totalement réel, $G$ l'ensemble des plongements de $K$ dans $\mathbb{C}$ et $\theta$ un entier algébrique dont le polynôme minimal sur $K$, noté $P$, est non réciproque. (Un polynôme $P$ de degré $d$ est dit réciproque si la fraction $P^{*} / P$ est constante, où le polynôme $P^{*}$ est défini par $P^{*}(z)=z^{d} \bar{P}(1 / z), \bar{P}$ étant le polynôme dont les coefficients sont les conjugués complexes des coefficients de $P$.)

Si $\theta$ est de module supérieur à 1 et si toutes les autres racines de $P$ sont de module inférieur à 1 alors l'équation

$$
z^{n} P(z)+\varepsilon P^{*}(z)=0, \quad \varepsilon= \pm 1,
$$

admet à partir d'un certain rang (dépendant de $\theta$ seulement) une seule racine $\tau_{n}$ hors du disque unité et une seule racine à l'intérieur du disque unité. De plus, la suite $\tau_{n}$ converge vers $\theta$.

Si $K$ est un corps quadratique imaginaire on peut prendre pour $\varepsilon$ une unité de $K$.

La preuve de ce résultat est analogue à celle donnée par Salem pour le $\operatorname{cas} K=\mathbb{Q}[4]$.

La méthode. Soit $\theta$ un $K$-nombre de Pisot et $\sigma \in G$. Le polynôme $\sigma P$ admet une seule racine $\theta_{\sigma}$ de module $>1$ et aucune racine de module 1 . De ce qui précède on déduit l'existence d'une suite d'entiers algébriques $\left(\tau_{\sigma, n}\right)_{n}$ convergeant vers $\theta_{\sigma}$ telle que les $\tau_{\sigma, n}$ lorsque $n$ est fixé et $\sigma$ parcourt $G$ soient racines d'un même polynôme unitaire à coefficients entiers rationnels :

$$
\prod_{\sigma \in G}\left(z^{n} \sigma P(z)+\varepsilon \sigma P^{*}(z)\right) .
$$

De plus, chaque $\tau_{\sigma, n}$ admet (à partir d'un certain rang) au moins un conjugué de module 1. La méthode consiste alors à appliquer la construction de Salem aux $K$-nombres de Pisot de mesure minimale. 
Commençons d'abord par donner une minoration de la mesure d'un polynôme non réciproque réductible sur certains corps de nombres primitifs. (Un corps de nombres $K$ est dit primitif s'il n'existe aucun corps intermédiaire entre $\mathbb{Q}$ et $K$.)

THÉORÈmE 1. Soient $K$ un corps de nombres totalement réel primitif de degré $d$ ou bien un corps quadratique de discriminant $D$ et $P$ le polynôme minimal sur $K$ d'un entier algébrique $\theta$. Si le polynôme minimal de $\theta$ sur $K$ est non réciproque et si pour tout plongement $\sigma$ de $K$ dans $\mathbb{C}$ les polynômes $P$ et $\sigma P$ sont premiers entre eux alors

$$
M(\theta)^{2(d-1)} \geq|D| / d^{d} .
$$

Preuve. Avec les notations précédentes, considérons la fonction non constante $f$ définie par

$$
f(z)=P(z) / P^{*}(z) .
$$

La fonction $f$ est méromorphe dans le disque unité avec $r \geq 0$ pôles, est de module 1 sur le cercle unité et admet un développement en série de Taylor au voisinage de l'origine à coefficients entiers du corps $K$ de la forme

$$
f(z)=\sum_{n \geq 0} u_{n} z^{n} .
$$

Comme le corps $K$ est ou bien totalement réel ou bien quadratique, la conjugaison complexe commute avec tout élément $\sigma$ de $G$ et par suite on a l'égalité

$$
(\sigma P)^{*}=\sigma P^{*},
$$

où $\sigma P$ (resp. $\sigma P^{*}$ ) désigne le polynôme dont les coefficients sont les conjugués par $\sigma$ des coefficients de $P$ (resp. de $P^{*}$ ).

Considérons alors la fonction $\sigma f$ définie par

$$
\sigma f(z)=\sigma P(z) / \sigma P^{*}(z),
$$

où $\sigma$ est un élément de $G$. La fonction $\sigma f$ est méromorphe dans le disque unité avec $r_{\sigma} \geq 0$ pôles, est de module 1 sur le cercle unité et admet un développement en série de Taylor au voisinage de l'origine de la forme

$$
\sigma f(z)=\sum_{n \geq 0} \sigma u_{n} z^{n},
$$

où $\sigma u_{n}$ désigne le conjugué par $\sigma$ de $u_{n}$.

Soit alors la fonction $\Delta$ définie par

$$
\Delta(z)=\prod_{\sigma \neq \phi}(\sigma f(z)-\phi f(z)), \quad(\sigma, \phi) \in G^{2} .
$$

Comme le polynôme $P$ est premier à tous ses conjugués, on déduit qu'il en est de même pour tout couple de polynômes $\sigma P$ et $\phi P$ si $\sigma \neq \phi$, et comme le 
polynôme minimal de $\theta$ sur $K$ est non réciproque on déduit que la fonction $\Delta$ n'est pas identiquement nulle; de plus, le corps $K$ étant primitif, on déduit l'existence d'un entier naturel $N$ (on choisit le plus petit) tel que l'entier $u_{N}$ engendre le corps $K$.

Soit $H$ la fonction définie par

$$
H(z)=\left(\Delta(z) / z^{N d(d-1)}\right)\left(\prod_{1 \leq i \leq k}\left(1-\theta_{i} z\right) /\left(z-\theta_{i}\right)\right)^{2(d-1)},
$$

où $\theta_{1}, \ldots, \theta_{k}$ désignent les conjugués de $\theta$ sur $\mathbb{Q}$ hors du disque unité.

La fonction $H$ est une fonction holomorphe sur un ouvert contenant le disque unité, est de même module que la fonction $\Delta$ sur le cercle unité, module qu'on peut majorer par $d^{d}$ grâce à l'inégalité de Hadamard. En outre, sa valeur à l'origine est

$$
H(0)=\left(\prod_{\sigma \neq \phi}\left(\sigma u_{N}-\phi u_{N}\right)\right) / M(\theta)^{2(d-1)} .
$$

Du principe du maximum on déduit $|H(0)| \leq d^{d}$, d'où le résultat.

\section{Cas quadratique imaginaire}

2.1. Soit $K=\mathbb{Q}(\sqrt{d})$ un corps quadratique et $\theta$ un $K$-nombre de Pisot de polynôme minimal $P$ (resp. $F$ ) sur $K$ (resp. sur $\mathbb{Q}$ ). De la définition 2 on déduit que soit $P=F$ et dans ce cas $\pm \theta$ est un nombre de Pisot, soit $P \neq F$ et dans ce cas $\theta$ n'est pas réel et admet deux conjugués sur $\mathbb{Q}$ de module $>1$.

Par la suite on va déterminer les valeurs possibles de $\theta$ lorsque $d<$ $0, M(\theta)<2$ et $P \neq F$; pour ceci on a besoin de certains lemmes que l'on applique plusieurs fois.

Lemme 1. Soient $\xi$ une unité non réelle d'un corps quadratique $K$ et $\theta$ un nombre réel. Alors on a équivalence entre les assertions suivantes:

(i) $|\theta|$ est un nombre de Pisot (resp. de Salem);

(ii) $\xi \theta$ est un $K$-nombre de Pisot (resp. de Salem) non réel.

Preuve. Ce cas n'a lieu que si $K=\mathbb{Q}(\sqrt{-1})$ ou bien $K=\mathbb{Q}(\sqrt{-3})$. Si $|\theta|$ est un nombre de Pisot (resp. de Salem) alors $\xi \theta$ est un élément primitif du corps $\mathbb{Q}(\xi, \theta)$ car les plongements de ce corps dans $\mathbb{C}$ transforment $\xi \theta$ en $\xi \theta, \bar{\xi} \theta$, ou bien en des conjugués de module inférieur à 1 , et comme $\xi$ est non réel on déduit $K \subseteq \mathbb{Q}(\xi, \theta)=\mathbb{Q}(\xi \theta)$, d'où le résultat.

Inversement, l'égalité $\xi \bar{\xi} \theta^{2}=\theta^{2}$ montre que $\theta$ est un entier algébrique de module $>1$ et comme $K(\xi \theta)=\mathbb{Q}(\xi \theta)=\mathbb{Q}(\xi, \theta)$, on déduit que si $\xi \theta$ est de degré $2 s$ sur $\mathbb{Q}$, alors $\theta$ est de degré $s$ sur $\mathbb{Q}$. 
Comme pour tout plongement $\sigma$ de $\mathbb{Q}(\xi \theta)$ dans $\mathbb{C}$ on a

$$
|\sigma(\xi \theta)|=|\sigma(\xi) \sigma(\theta)|=|\sigma(\theta)|,
$$

en considérant les prolongements de l'identité de $K$ on déduit le résultat.

Lemme 2 [10]. Soit $f$ une fonction méromorphe non constante admettant $k$ pôles à l'intérieur du disque unité, vérifiant $|f(z)| \leq 1$ sur le cercle unité et admettant un développement de Taylor au voisinage de l'origine de la forme

$$
f(z)=1+u_{k} z^{k}+\ldots
$$

Alors $u_{k}$ est non nul.

Lemme 3 (Schur). Soit $f$ une fonction holomorphe sur un ouvert contenant le disque unité telle que $|f(z)| \leq 1$ sur le cercle unité. Une telle fonction est appelée fonction de Schur. Si $f$ n'est pas constante, alors la fonction $f_{1}$ définie par

$$
f_{1}(z)=\frac{f(z)-f(0)}{z(1-\overline{f(0)} f(z))}
$$

est aussi une fonction de Schur.

LEMme 4 (Schur). Soit $f$ une fonction de Schur admettant un développement en série de Taylor au voisinage de l'origine de la forme $\sum_{n \geq 0} u_{n} z^{n}$. Alors $\left|u_{1}\right| \leq 1-\left|u_{0}^{2}\right|$.

Lemme 5. Soit $f$ une fonction méromorphe sur un ouvert contenant le disque unité n'ayant qu'un pôle simple de module inférieur à 1 , vérifiant $|f(z)|=1$ sur le cercle unité et admettant un développement en série de Taylor au voisinage de l'origine à coefficients entiers d'un corps quadratique imaginaire $\mathbb{Q}(\sqrt{d})$ de la forme

$$
f(z)=1+z+z^{2}+u_{3} z^{3}+\ldots+u_{n} z^{n}+\ldots
$$

Alors

(a) si $d=-1$ alors ou bien $u_{n} \in \mathbb{Z}$ pour tout $n$, ou bien

$$
f(z)=\frac{1-z^{2} \pm i z^{n}\left(1+z-z^{2}\right)}{\left(1-z-z^{2}\right) \pm i z^{n}\left(1-z^{2}\right)}
$$

$i$ désignant la racine carré de -1 ;

(b) si $d=-3$ alors ou bien $u_{n} \in \mathbb{Z}$ pour tout $n$, ou bien il existe une unité non réelle $j$ dans $\mathbb{Q}(\sqrt{d})$ telle que

$$
f(z)=\frac{1-z^{2} \pm j z^{n}\left(1+z-z^{2}\right)}{\left(1-z-z^{2}\right) \pm j z^{n}\left(1-z^{2}\right)} ;
$$

(c) si $d \neq-1$ et $d \neq-3$, alors $u_{n} \in \mathbb{Z}$ pour tout $n$.

Preuve. La preuve de ce résultat est identique au cas où $f$ admet un développement en série de Taylor à coefficients dans $\mathbb{Z}$ [7]. 
ThÉORÈme 2. Soient $K=\mathbb{Q}(\sqrt{d})$ un corps quadratique imaginaire et $\theta$ un $K$-nombre de Pisot non réel de polynôme minimal $\operatorname{Irr}(\theta, K, z)$ (resp. $\operatorname{Irr}(\theta, \mathbb{Q}, z))$ sur $K($ resp. $\mathbb{Q})$ et de mesure $M(\theta)$.

(a) Si $d=-7$ alors $M(\theta) \geq 2$ sauf si

$$
\begin{aligned}
& \operatorname{Irr}( \pm \theta, K, z)=z^{2}+(1 \pm \sqrt{-7}) z / 2-1 \\
& \operatorname{Irr}( \pm \theta, \mathbb{Q}, z)=z^{4}+z^{3}-z+1 \text { et } M(\theta)=1.8832 \ldots ;
\end{aligned}
$$

(b) si $d=-1$ alors $M(\theta) \geq 2$ sauf si

$$
\begin{aligned}
& \operatorname{Irr}( \pm \theta, K, z)=z^{3}+z+i, \\
& \operatorname{Irr}(\theta, \mathbb{Q}, z)=z^{6}+2 z^{4}+z^{2}+1 \text { et } M(\theta)=\theta_{0}^{2}=1.7548 \ldots, \\
& \operatorname{Irr}(\xi \theta, K, z)=z^{3}-z^{2} \pm i, \\
& \operatorname{Irr}(\xi \theta, \mathbb{Q}, z)=z^{6}-2 z^{5}+z^{4}+1 \text { et } M(\theta)=1.8977 \ldots, \\
& \operatorname{Irr}( \pm \theta, K, z)=z^{4}+i z^{3}-1, \\
& \operatorname{Irr}(\theta, \mathbb{Q}, z)=z^{8}+z^{6}-2 z^{4}+1 \text { et } M(\theta)=\theta_{1}^{2}=1.9051 \ldots, \\
& \operatorname{Irr}(\xi \theta, K, z)=z^{3} \pm i\left(z^{2}+z\right)-z-1, \\
& \operatorname{Irr}(\xi \theta, \mathbb{Q}, z)=z^{6}-z^{4}+2 z^{2}+2 z+1 \text { et } M(\theta)=1.9922 \ldots,
\end{aligned}
$$

où $\xi$ est l'une des puissances de la racine primitive quatrième de l'unité $i$ et où $\theta_{0}$ et $\theta_{1}$ désignent les deux plus petits nombres de Pisot;

(c) si $d=-3$ alors $M(\theta) \geq 2$ sauf si

$$
\begin{aligned}
& \operatorname{Irr}(\eta \theta, K, z)=z^{2}+j z-1 \text { ou bien } \operatorname{Irr}(\eta \theta, K, z)=z^{2}+\bar{j} z-1, \\
& \operatorname{Irr}(\eta \theta, \mathbb{Q}, z)=z^{4}+z^{3}-z^{2}-z+1 \text { et } M(\theta)=1.7220 \ldots, \\
& \operatorname{Irr}( \pm \theta, K, z)=z^{3}+j z+1 \text { ou bien } \operatorname{Irr}( \pm \theta, K, z)=z^{3}+\bar{j} z+1, \\
& \operatorname{Irr}( \pm \theta, \mathbb{Q}, z)=z^{6}+z^{4}+2 z^{3}+z^{2}+z+1 \text { et } M(\theta)=\theta_{0}^{2}=1.7548 \ldots, \\
& \operatorname{Irr}(\eta \theta, K, z)=z^{3}-j z^{2}-z-\bar{j} \text { ou bien } \operatorname{Irr}(\eta \theta, K, z)=z^{3}-\bar{j} z^{2}-z-j, \\
& \operatorname{Irr}(\eta \theta, \mathbb{Q}, z)=z^{6}-z^{5}-z^{4}+z+1 \text { et } M(\theta)=1.8378 \ldots, \\
& \operatorname{Irr}( \pm \theta, K, z)=z^{4}-j z^{3}+j \text { ou bien } \operatorname{Irr}( \pm \theta, K, z)=z^{4}-\bar{j} z^{3}+\bar{j}, \\
& \operatorname{Irr}( \pm \theta, \mathbb{Q}, z)=z^{8}-z^{7}+z^{6}+z^{4}-2 z^{3}+1 \text { et } M(\theta)=\theta_{1}^{2}=1.9051 \ldots,
\end{aligned}
$$

où $\eta$ est l'une des puissances de la racine primitive sixième de l'unité $j=$ $(1+\sqrt{-3}) / 2$

(d) si $d \neq-1, d \neq-3$ et $d \neq-7$ alors $M(\theta) \geq 2$.

Preuve. Soit $\theta$ un $\mathbb{Q}(\sqrt{d})$-nombre de Pisot non réel de mesure $<2$ et de polynôme minimal $P$ sur $\mathbb{Q}(\sqrt{d})$.

Comme $P(0)$ est un entier de $\mathbb{Q}(\sqrt{d})$ vérifiant $|P(0)| \leq|\theta|<\sqrt{2}$, il est alors de module 1 .

Supposons d'abord que le polynôme minimal de $\theta$ sur $\mathbb{Q}$ soit non réciproque. Du théorème 1 on déduit que la valeur absolue du discriminant du 
corps $\mathbb{Q}(\sqrt{d})$ est inférieure à 16 et comme il y a une bijection entre les corps quadratiques et leurs discriminants on déduit un nombre fini de valeurs possibles pour $d$. Considérons alors la fraction rationnelle

$$
f(z)=\overline{P(0)} P(z) / P^{*}(z) .
$$

La fonction $f$ est méromorphe dans le disque unité, admet un pôle simple $1 / \bar{\theta}$ à l'intérieur du disque unité, est de module 1 sur le cercle unité et admet un développement en série de Taylor au voisinage de l'origine à coefficients entiers de $\mathbb{Q}(\sqrt{d})$ de la forme

$$
f(z)=1+u_{1} z+u_{2} z^{2}+\ldots+u_{n} z^{n}+\ldots
$$

Montrons en premier lieu que $\left|u_{1}\right|=1$ et que $M(\theta)>\theta_{\infty}=(1+\sqrt{5}) / 2$.

Soit $F$ la fonction de Schur non constante définie par

$$
F(z)=(1-\bar{\theta} z) f(z) /(z-\theta) .
$$

La première transformée de Schur de $F$,

$$
F_{1}(z)=\frac{F(z)-F(0)}{z(1-\overline{F(0)} F(z))},
$$

est aussi une fonction de Schur d'après le lemme 3. Le lemme 4 appliqué à la fonction $F_{1}$ donne

$$
\left|u_{1} \theta+1-\right| \theta^{2}|| \leq|\theta|^{2}-1
$$

d'où

$$
\left|u_{1}\right| \leq 2\left(|\theta|^{2}-1\right) /|\theta|<\sqrt{2} .
$$

La dernière inégalité est due au fait que la fonction $\left(x^{2}-1\right) / x$ est strictement croissante sur $] 0, \infty\left[\right.$. On déduit alors que $\left|u_{1}\right| \in\{0,1\}$.

Le lemme 2 appliqué à la fonction $f$ montre que $u_{1} \neq 0$, la fonction $f$ n'étant pas constante (car les polynômes $P$ et $P^{*}$ sont premiers entre eux).

Remarquons que (1) entraîne que $u_{1}=0$ si $|\theta|<(1+\sqrt{17}) / 4$.

Par conséquent, on a le résultat suivant :

$$
M(\theta) \geq((1+\sqrt{17}) / 4)^{2}>\theta_{\infty} \quad \text { et } \quad\left|u_{1}\right|=1 .
$$

Pour la suite, on distingue quatre cas suivant les valeurs de $d$.

1. $d \equiv 2$ ou $d \equiv 3$ modulo $4, d \neq-1$. Dans ce cas l'égalité $\left|u_{1}\right|=1$ entraîne que $u_{1}= \pm 1$.

Il suffit d'étudier le cas $u_{1}=1$. En effet, si $\theta$ est un $\mathbb{Q}(\sqrt{d})$-nombre de Pisot associé à $f(z),-\theta$ l'est aussi, est associé à $f(-z)$ et est de même mesure que $\theta$. 
Considérons la fonction de Schur suivante :

$$
G(z)=\frac{(1-\theta z)(1-\bar{\theta} z)(f(z)-\bar{f}(z))}{2 z^{2}(\theta-z)(\bar{\theta}-z)}, \quad \text { où } \quad \bar{f}(z)=\frac{\bar{P}(z)}{\bar{P}^{*}(z)} .
$$

Le principe du maximum appliqué à la fonction non constante $G$ donne alors

$$
\left|u_{2}-\bar{u}_{2}\right| \leq 2|\theta|^{2}<4
$$

d'où on déduit l'inégalité

$$
\left|b_{2}\right|<2 / \sqrt{-d} \text { avec } u_{2}=a_{2}+i b_{2} \sqrt{-d} \text { et } b_{2} \in \mathbb{Z} .
$$

Si $d \leq-5$ alors $b_{2}=0$ et $u_{2}=\bar{u}_{2}$. Le lemme 4 appliqué à la première transformée de Schur de $G$,

$$
G_{1}(z)=G(z) / z,
$$

donne aussi que $u_{3}=\bar{u}_{3}$; ainsi de suite on obtient $u_{n}=\bar{u}_{n}$ pour tout $n$, ce qui entraîne l'égalité des deux fractions $f$ et $\bar{f}$, ce qui est impossible puisque le polynôme minimal de $\theta$ sur $\mathbb{Q}$ est non réciproque.

Si $d=-2$, considérons la fonction de Schur $H$ définie par

$$
H(z)=f(z) f(-z)\left(1-\bar{\theta}^{2} z^{2}\right) /\left(\theta^{2}-z^{2}\right) .
$$

Sa première transformée de Schur étant nulle à l'origine, on déduit que sa deuxième transformée de Schur vaut

$$
H_{2}(z)=\frac{H(z)-H(0)}{z^{2}(1-\overline{H(0)} H(z))} .
$$

Le lemme 4 appliqué à la fonction de Schur $H_{2}$ donne

$$
\left|\left(2 u_{2}-1\right) \theta^{2}+1-\right| \theta^{4}||<|\theta|^{4}-1 .
$$

On déduit alors l'inégalité

$$
\left|2 u_{2}-1\right| \leq 2\left(|\theta|^{4}-1\right) /|\theta|^{2}<3 \quad \text { si }|\theta|<\sqrt{2} .
$$

La dernière inégalité est due au fait que la fonction $\left(x^{2}-1\right) / x$ est strictement croissante sur $] 0, \infty\left[\right.$. On déduit alors que $u_{2} \in\{0,1\}$.

Cependant aucun de ces deux cas ne peut avoir lieu. En effet, d'une part, le lemme 2 appliqué à la fonction non constante $(f(z)+f(-z)) / 2$ montre que $u_{2} \neq 0$; d'autre part, le lemme 5 appliqué à la fonction $f$ montre que $f$ admet un développement en série de Taylor à coefficients dans $\mathbb{Z}$, ce qui n'est pas le cas puisque les fonctions $f$ et $\bar{f}$ ne sont pas identiques.

On conclut donc pour ce cas que $M(\theta) \geq 2$.

2. $d \equiv 1$ modulo $4, d \neq-3$. Dans ce cas l'égalité $\left|u_{1}\right|=1$ entraîne l'égalité $u_{1}= \pm 1$. Comme précédemment on peut se restreindre au cas $u_{1}=1$.

On considère la fonction $H$ comme dans le cas $d=-2$; les mêmes calculs montrent que $u_{2} \in\{0,1,(1 \pm i \sqrt{7}) / 2\}$ et de la même manière on montre que les 2 premiers cas ne peuvent pas avoir lieu. 
Si $d=-7$ et $u_{2}=(1 \pm i \sqrt{7}) / 2$, le lemme 2 appliqué à la fonction $f(z) \bar{f}(-z)$ montre que cette fonction est constante égale à 1 , c'est-à-dire que $\theta$ et $-1 / \bar{\theta}$ sont conjugués, cas que l'on étudiera séparément.

Ceci achève la preuve $\mathrm{du}(\mathrm{d})$.

3. $d=-1$. L'égalité $\left|u_{1}\right|=1$ entraîne que $u_{1}= \pm 1, \pm i$; dans ce cas aussi on peut supposer $u_{1}=1$. En effet, si $u_{1}=-1$ on prend $f(-z)$ au lieu de $f(z)$ puisque le nombre $-\theta$ est un $\mathbb{Q}(i)$-nombre de Pisot de même mesure. Si $u_{1}=i$ on prend $f(-i z)$ au lieu de $f(z)$; en effet, d'après le lemme 1 le nombre $i \theta$ est soit un $\mathbb{Q}(i)$-nombre de Pisot de même mesure que $\theta$, soit un nombre de Pisot de mesure $<\sqrt{2}$; mais les nombres de Pisot de mesure $<\sqrt{2}$ sont connus et sont soit $\theta_{0}=1.32 \ldots$ soit $\theta_{1}=1.38 \ldots$

On a le même résultat si $u_{1}=-i$.

On considère la fonction $H$ comme pour les cas précédents et on obtient que $u_{2} \in\{0,1, \pm i, 1 \pm i\}$. On montre que $u_{2} \neq 0$ de la même manière que pour les cas précédents.

- $u_{2}= \pm i$. Le lemme 2 appliqué à la fonction $(f(z)+\bar{f}(-z)) / 2$ montre que cette fonction est nécessairement constante; on en déduit que $\bar{\theta}=-\theta$ et par le lemme 1 que $\pm \theta / i$ est un nombre de Pisot inférieur à $\sqrt{2}$, donc égal à $\theta_{0}$ ou $\theta_{1}$.

- $u_{2}=1$. Le lemme 5 appliqué à la fonction $f$ donne dans ce cas que $P(z)$ est de la forme

$$
z^{n}\left(z^{2}-z-1\right) \pm i\left(z^{2}-1\right), \quad n \geq 1 .
$$

Les racines $\left(\theta_{n}\right)_{n}$ hors du disque unité de ces polynômes sont des $\mathbb{Q}(i)$ nombres de Pisot de mesure supérieure à 2 qui convergent vers le nombre d'or $\theta_{\infty}$ racine $>1$ de $z^{2}-z-1$. En effet, pour $|z|=\sqrt{2}$ et $n>2$ on a

$$
\begin{aligned}
\left|z^{n}\left(z^{2}-z-1\right)\right|^{2}-\left|z^{2}-1\right|^{2} & =2^{n} 7-5-2^{n}(z+\bar{z})+\left(1-2^{n}\right)\left(z^{2}+\bar{z}^{2}\right) \\
& >2^{n}(3-2 \sqrt{2})-1>0 .
\end{aligned}
$$

Comme $\theta_{\infty}>\sqrt{2}$, le théorème de Rouché entraîne que $\left|\theta_{n}\right| \geq \sqrt{2}$ si $n \geq 3$. Pour $n \leq 2$, le calcul direct montre que $\left|\theta_{n}\right|^{2} \geq 2$.

- $u_{2}=1 \pm i$. On peut se limiter au cas où $u_{2}=1+i$, le cas $u_{2}=1-i$ conduisant à la fraction conjuguée de $f$. Considérons alors la fonction $g$ définie par

$$
g(z)=\frac{\left(z^{2}+(1+i) z-1\right) f(z)-\left(z^{2}+i z-1\right)}{\left(z^{2}-(1-i) z-1\right)-f(z)\left(z^{2}+i z-1\right)} .
$$

Comme dans la preuve du lemme 5 on montre que la fonction $g$ est de Schur. Si $\sum_{n \geq 0} \alpha_{n} z^{n}$ désigne son développement en série de Taylor au voisinage 
de l'origine, on déduit de la formule intégrale de Cauchy

$$
\sum_{n \geq 0}\left|\alpha_{n}\right|^{2} \leq 1
$$

et par suite $g(z)= \pm z^{n}$ ou $g(z)= \pm i z^{n}$; on déduit alors les 4 formes possibles pour $P$ :

1) $z^{n}\left(z^{2}-(1-i) z-1\right)+\left(z^{2}+i z-1\right), n \geq 1$,

2) $z^{n}\left(z^{2}-(1-i) z-1\right)-\left(z^{2}+i z-1\right), n \geq 1$,

3) $z^{n}\left(z^{2}-(1-i) z-1\right)+i\left(z^{2}+i z-1\right), n \geq 1$,

4) $z^{n}\left(z^{2}-(1-i) z-1\right)-i\left(z^{2}+i z-1\right), n \geq 1$.

Toutes ces familles donnent lieu à des suites de $\mathbb{Q}(i)$-nombres de Pisot convergeant vers le $\mathbb{Q}(i)$-nombre de Pisot racine de module $1.700 \ldots$ du polynôme $z^{2}-(1-i) z-1$.

Pour $n \geq 4$, ces $\mathbb{Q}(i)$-nombres de Pisot sont de mesure $\geq 2$. En effet, si $z=\sqrt{2} e^{i \alpha}$ où $\alpha \in[0,2 \pi]$ alors

$$
\left|z^{2}+i z-1\right|^{2}=7+6 \sqrt{2} \sin (\alpha)-4 \cos (2 \alpha)=R(\alpha)
$$

et

$$
\left|z^{2}-(1-i) z-1\right|^{2}=R(\alpha)+2-2 \sqrt{2} \cos (\alpha)=L(\alpha) .
$$

On déduit que si $\pi / 4<\alpha<7 \pi / 4$ alors $L(\alpha)>R(\alpha)$. De même, si $0 \leq \alpha \leq$ $\pi / 4$ et $n \geq 1$ alors

$$
2^{n} L(\alpha)-R(\alpha) \geq R(\alpha)+4-4 \sqrt{2} \cos (\alpha)>7-4 \sqrt{2}>0 .
$$

Les fonctions $L$ et $R$ sont croissantes sur l'intervalle [ $-13 \pi / 90,0]$; on déduit alors pour $n \geq 4$ les inégalités suivantes :

$$
L(\alpha) \geq L(-13 \pi / 90)>0.25>3 / 2^{n}=R(0) / 2^{n} \geq R(\alpha) / 2^{n} .
$$

Sur l'intervalle $[-\pi / 4,-3 \pi / 20]$ la fonction $L$ est décroissante et la fonction $R$ est majorée par 4 ; on déduit alors pour $n \geq 4$ les inégalités suivantes :

$$
L(\alpha) \geq L(-3 \pi / 20)>0.25 \geq 4 / 2^{n} \geq R(\alpha) / 2^{n} .
$$

Enfin, si $-3 \pi / 20 \leq \alpha \leq-13 \pi / 90$ alors la fonction $R$ est majorée par 1 et la fonction $L$ est minorée par 0.14 ; on déduit alors l'inégalité $2^{n} L(\alpha)>R(\alpha)$ pour tout $n \geq 3$.

On déduit que si $n \geq 4$ alors $2^{n} L(\alpha)>R(\alpha)$; et le théorème de Rouché entraîne le résultat.

Pour $n \leq 4$, le calcul direct montre que seuls les cas où $n=1$ dans les familles 1) $\left(\left|\theta_{1}\right|=1.4114 \ldots\right)$ et 4$)\left(\left|\theta_{1}\right|=1.3775 \ldots\right)$ donnent des mesures $<2$.

On obtient alors respectivement les deux $\mathbb{Q}(i)$-nombres de Pisot de mesure $1.9922 \ldots$ et $1.8977 \ldots$ et de polynômes minimaux sur $\mathbb{Q}$

$$
z^{6}-z^{4}+2 z^{2}+2 z+1 \quad \text { et } z^{6}-2 z^{5}+z^{4}+1 .
$$


4. $d=-3$. Comme $u_{1}$ est de module 1 , le lemme 1 montre qu'on peut supposer $u_{1}=1$. On considère la fonction $H$ comme dans les cas précédents et on obtient alors

$$
u_{2} \in\{0,1,1+j, 1+\bar{j}, \pm j, \pm \bar{j}\} .
$$

De la même manière que pour les cas précédents on montre que $u_{2} \neq 0$.

- $u_{2}=1$. Le lemme 5 appliqué à la fonction $f$ donne pour valeurs possibles de $P$,

$$
z^{n}\left(z^{2}-z-1\right) \pm j\left(1-z^{2}\right) \quad \text { ou } \quad z^{n}\left(z^{2}-z-1\right) \pm \bar{j}\left(1-z^{2}\right) .
$$

On obtient ainsi deux suites de $\mathbb{Q}(\sqrt{-3})$-nombres de Pisot convergeant vers le nombre d'or $\theta_{\infty}$.

Seul le premier terme de la première suite est de mesure inférieure à 2 , soit $\theta_{1}$ racine de $z^{3}-j z^{2}-z-\bar{j}$ de module $1.3556 \ldots$; la preuve ici est la même que celle pour le cas où $d=-1$ et $u_{2}=1$. On obtient ainsi le $\mathbb{Q}(\sqrt{-3})$-nombre de Pisot de mesure $1.8378 \ldots$ et de polynôme minimal sur le corps des rationnels $z^{6}-z^{5}-z^{4}+z+1$.

- $u_{2}=j$ ou $u_{2}=\bar{j}$. Le lemme 2 appliqué à la fonction $f(z) \bar{f}(-z)$ montre que cette fonction est nécessairement constante et par suite que $-1 / \theta$ est racine de $P$; ce cas sera étudié séparément.

- $u_{2}=-j$. Montrons que ce cas ne peut pas avoir lieu. Considérons la fonction auxiliaire $f_{0}$ définie par

$$
f_{0}(z)=\left(z^{2}+j z-1\right) /\left(z^{2}+(1+j) z-1\right) ;
$$

$f_{0}$ admet un pôle simple à l'intérieur du disque unité $1 / \varrho_{0}\left(\left|\varrho_{0}\right|=2.07 \ldots\right)$, vérifie $\left|f_{0}(z)\right|<1$ si $|z|=1$ et admet un développement de Taylor au voisinage de l'origine de la forme

$$
f_{0}(z)=1+z+(1+j) z^{2}+\ldots
$$

Le lemme 2 appliqué à la fonction $f_{0}(z) f(-z)$ montre que ce cas ne peut pas avoir lieu.

De même le cas $u_{2}=-\bar{j}$ ne peut pas avoir lieu puisqu'il conduit à la fraction conjuguée de $f$.

- $u_{2}=1+j$. Considérons la fonction $g$ définie par $g(z)=N(z) / D(z)$, où

$$
\begin{aligned}
& N(z)=\left(z^{2}+(1+j) z-1\right) f(z)-\left(z^{2}+j z-1\right), \\
& D(z)=\left(z^{2}-(1+\bar{j}) z-1\right)-\left(z^{2}-\bar{j} z-1\right) f(z) .
\end{aligned}
$$

Alors $g$ est une fonction de Schur et de la même manière que pour le cas $d=-1$ et $u_{2}=1+i$ on déduit que $P$ a l'une des 6 formes possibles :

$$
z^{n}\left(z^{2}-(1+\bar{j}) z-1\right) \pm \xi\left(z^{2}+j z-1\right), \quad n \geq 1,
$$

$\xi$ désignant l'une des 6 unités de $\mathbb{Q}(\sqrt{-3})$. 
Chacune de ces familles donne lieu à une suite de $\mathbb{Q}(\sqrt{-3})$-nombres de Pisot convergeant vers le $\mathbb{Q}(\sqrt{-3})$-nombre de Pisot racine du polynôme $z^{2}-(1+\bar{j}) z-1$ et de module $2.07 \ldots$; tous ces $\mathbb{Q}(\sqrt{-3})$-nombres de Pisot sont de mesure $\geq 2$.

En effet, si $z=\sqrt{2} e^{i \alpha}$ où $\alpha \in[0,2 \pi]$ alors

$$
\left|z^{2}-1+j z\right|^{2}=7-4 \cos (2 \alpha)+\sqrt{2} \cos (\alpha)+3 \sqrt{6} \sin (\alpha)=R(\alpha)
$$

et

$$
\left|z^{2}-1-(1+\bar{j}) z\right|^{2}=R(\alpha)+4-4 \sqrt{2} \cos (\alpha)=L(\alpha) .
$$

On déduit que si $\pi / 4<\alpha<7 \pi / 4$ alors $L(\alpha)>R(\alpha)$. De même, si $0 \leq \alpha \leq$ $\pi / 4$ et $n \geq 1$ alors

$2^{n} L(\alpha)-R(\alpha) \geq 15-4 \cos (2 \alpha)+3 \sqrt{6} \sin (\alpha)-7 \sqrt{2} \cos (\alpha) \geq 11-7 \sqrt{2}>0$.

Sur l'intervalle $[-\pi / 10,0]$, la fonction $L$ est croissante et la fonction $R$ est majorée par 5.2; on déduit alors pour $n \geq 2$ les inégalités suivantes :

$$
L(\alpha) \geq L(-\pi / 10)>1.45>(5.2) / 2^{n} \geq R(\alpha) / 2^{n} .
$$

Sur l'intervalle $[-\pi / 4,-\pi / 7$, la fonction $L$ est décroissante et la fonction $R$ est majorée par 5.1 ; on déduit alors pour $n \geq 2$ les inégalités suivantes :

$$
L(\alpha) \geq L(-\pi / 7)>1.5>(5.1) / 2^{n}>R(\alpha) / 2^{n} .
$$

Enfin, sur l'intervalle $[-\pi / 7,-\pi / 10]$ la fonction $R$ est majorée par 4 et la fonction $L$ est minorée par 0.5 ; on déduit alors pour $n \geq 3$ l'inégalité $2^{n} L(\alpha)>R(\alpha)$, et le théorème de Rouché entraîne le résultat. Pour $n \leq 2$, le calcul direct montre que ce cas ne peut pas avoir lieu.

Le cas $u_{2}=1+\bar{j}$ conduit aux polynômes conjugués et les mesures sont les mêmes.

Supposons enfin que $P(z)=(z-\theta)(z+1 / \theta)$ ou $P(z)=(z-\theta)(z+1 / \bar{\theta})$ ou $P(z)=(z-\theta)(z-1 / \theta)$.

Le degré de $P$ ainsi que ses coefficients (entiers d'un corps quadratique imaginaire) sont bornés, on déduit alors un nombre fini de valeurs possibles; les calculs montrent que deux cas seulement peuvent avoir lieu :

- $d=-3$. Pour ce cas le polynôme $P$ prend douze valeurs différentes; en changeant au besoin $P(z)$ en $P(-z)$ ou bien $P(z)$ en $\bar{P}(z)$ on peut se restreindre aux valeurs suivantes :

$$
z^{2}+j z-1, \quad z^{2}-j z+j, \quad z^{2}-z+j .
$$

Les mesures de ces polynômes sont égales et valent $1.7220 \ldots$ 
- $d=-7$. Pour ce cas le polynôme $P$ prend 4 valeurs différentes. Pour la même raison que précédemment on peut se restreindre à la valeur suivante :

$$
z^{2}+(1+i \sqrt{7}) z / 2-1 .
$$

La mesure de ce polynôme est égale à $1.8832 \ldots$

Il ne reste donc plus qu'à rajouter les $\mathbb{Q}(i)$-nombres de Pisot $\pm i \theta_{0}$ et $\pm i \theta_{1}$ et les $\mathbb{Q}(\sqrt{-3})$-nombres de Pisot $\pm j \theta_{0}, \pm j \theta_{1}, \pm \bar{j} \theta_{0}, \pm \bar{j} \theta_{1}$, où $\theta_{0}$ et $\theta_{1}$ désignent les nombres de Pisot inférieurs à $\sqrt{2}$.

Ceci achève la preuve du théorème.

THÉORÈme 3. Les valeurs de $M(\theta)$ inférieures à 2 de l'énoncé du théorème 2 sont des points d'accumulation de l'ensemble des mesures des entiers algébriques.

Preuve. La construction de Salem montre que si $\theta$ est un $\mathbb{Q}(\sqrt{d})$ nombre de Pisot non réel $(d<0)$ de polynôme minimal $P$ sur $\mathbb{Q}(\sqrt{d})$ non réciproque alors l'équation

$$
z^{n} P(z)+\xi P^{*}(z)=0,
$$

où $\xi$ désigne une unité de $\mathbb{Q}(\sqrt{d})$, admet pour $n$ assez grand comme racine hors du disque unité un $\mathbb{Q}(\sqrt{d})$-nombre de Salem $\tau_{n}$. De plus, la suite $\left(\tau_{n}\right)_{n}$ converge vers $\theta$.

Comme $\theta$ n'est pas réel, en considérant l'équation conjuguée complexe de $(*)$ on déduit $\lim M\left(\tau_{n}\right)=M(\theta)$, d'où le résultat.

2.2. Application à la détermination de polynômes réciproques de petites mesures. Avec les notations du théorème 3 et de sa preuve, l'équation

$$
z^{n} P(z)+\xi P^{*}(z)=0
$$

admet à partir d'un certain rang comme racine hors du disque unité un $\mathbb{Q}(\sqrt{d})$-nombre de Salem non réel $\tau_{n}$. De plus, $\lim M\left(\tau_{n}\right)=M(\theta) \geq m=$ $1.722 \ldots$, où $m$ désigne la plus petite mesure des $\mathbb{Q}(\sqrt{d})$-nombres de Pisot.

On déduit alors que si on fixe une borne supérieure $A<m$ pour les $M\left(\tau_{n}\right)$ on obtient au plus un nombre fini de valeurs des $\tau_{n}$.

Le lemme suivant montre que l'équation précèdente admet pour $n \geq 16$, pour $P$ l'un quelconque des polynômes de l'énoncé du théorème 2 et pour $\xi$ une unité quelconque du corps auquel appartiennent les coefficients de $P$, une racine hors du disque unité de valeur absolue $>1.3$.

On déduit alors que $\tau_{n}$ est ou bien non réel de mesure supérieure à 1.69 ou bien réel de mesure supérieure à 1.3 .

Lemme 6. Soient $\theta$ un $\mathbb{Q}(\sqrt{d})$-nombre de Pisot non réel de mesure $<2$ et de polynôme minimal $P$ sur $\mathbb{Q}(\sqrt{d}), n$ un entier naturel $\geq 16$ et $\xi$ est une 
unité de $\mathbb{Q}(\sqrt{d})$. Alors le polynôme $Q_{n}$ défini par:

$$
Q_{n}(z)=z^{n} P(z)+\xi P^{*}(z)
$$

admet une racine $\tau_{n}$ telle que $\left|\tau_{n}\right| \geq 1.3$.

Preuve. Soit $\theta$ un tel $\mathbb{Q}(\sqrt{d})$-nombre de Pisot; d'après le théorème 2 on a

$$
|\theta|>\sqrt{1.722}>1.3
$$

et l'on peut vérifier par un simple calcul que $\theta$ est sans conjugué sur le cercle $|z|=1 / 1.3$.

Si $\alpha$ est un conjugué de $\theta$ de module $<1$ au-dessus du corps $\mathbb{Q}(\sqrt{d})$ alors

$$
|z-\alpha|^{2}-|1-\bar{\alpha} z|^{2}=\left(|z|^{2}-1\right)\left(1-|\alpha|^{2}\right) ;
$$

on déduit alors pour $|z|=1.3$ l'inégalité

$$
\left|P(z) / \bar{P}^{*}(z)\right|>|(z-\theta) /(1-\bar{\theta} z)| .
$$

De manière identique à la preuve de la construction de Salem [4] on a

$$
|(z-\theta) /(1-\bar{\theta} z)|^{2} \geq g(|\theta|)=\left(|\theta|^{2}-2.6|\theta|+1.69\right) /\left(1.69|\theta|^{2}-2.6|\theta|+1\right) .
$$

Comme la fonction $g$ est croissante sur l'intervalle $[\sqrt{1.722}, \sqrt{2}]$, on déduit l'inégalité

$$
|(z-\theta) /(1-\bar{\theta} z)|^{2}>g(\sqrt{1.722})>0.0003,
$$

et par suite pour $|z|=1.3$ et $n \geq 16$ on a

$$
\left|z^{n} P(z) / \bar{P}^{*}(z)\right|^{2} \geq(1.69)^{n} \cdot 0.0003>1,
$$

et comme le polynôme $P$ admet une racine $\theta$ de module supérieur à 1.3 , le théorème de Rouché entraîne le résultat.

On peut donc déterminer tous les $\mathbb{Q}(\sqrt{d})$-nombres de Pisot non réels de mesure $<1.69$ ainsi que les nombres de Salem de mesure $<1.3$ de cette construction en affectant à $P$ les valeurs de l'énoncé du théorème 2 , à $n$ les entiers de 1 à 16 et à $\xi$ les unités du corps correspondant.

Les calculs ci-dessous montrent qu'on n'obtient que des $\mathbb{Q}(\sqrt{d})$-nombres de Salem non réels.

Pour chaque valeur de $P$ on précise dans l'ordre suivant et sur la même ligne du tableau 1 l'unité $\xi$, l'entier $n$, le degré du polynôme minimal sur $\mathbb{Q}$ du $\mathbb{Q}(\sqrt{d})$-nombre de Salem et enfin sa mesure.

Dans le cas où $\left|\tau_{n}\right|$ est un nombre de Salem (lemme 1), on note $\sigma_{k}^{2}$ la mesure de $\tau_{n}, \sigma_{k}$ désignant le $k$-ième plus petit nombre de Salem connu selon la notation de Boyd [5]. 
Tableau 1

\begin{tabular}{|c|c|c|c|c|c|}
\hline$d$ & $P(z)$ & & & & \\
\hline \multirow[t]{2}{*}{-7} & $z^{2}+(1+\sqrt{-7}) z / 2-1$ & 1 & 3 & 10 & $1.5817 \ldots$ \\
\hline & & 1 & 5 & 14 & $1.5671 \ldots$ \\
\hline \multirow[t]{10}{*}{-1} & $z^{3}+z+i$ & +1 & 11 & 16 & $\sigma_{22}^{2}=1.6400 \ldots$ \\
\hline & & -1 & 09 & 20 & $\sigma_{7}^{2}=1.5138$ \\
\hline & & $-i$ & 10 & 20 & $\sigma_{19}^{2}=1.5907 \ldots$ \\
\hline & & $+i$ & 08 & 20 & $\sigma_{1}^{2}=1.3836$ \\
\hline & & $+i$ & 12 & 20 & $\sigma_{38}^{2}=1.6731 \ldots$ \\
\hline & $z^{3}-z^{2}+i$ & 1 & 6 & 16 & $1.5771 \ldots$ \\
\hline & $z^{3}+i z^{2}-(1-i) z-1$ & 1 & 4 & 12 & $1.6385 \ldots$ \\
\hline & $z^{4}-i z^{3}-1$ & $+i$ & 7 & 20 & $\sigma_{5}^{2}=1.4796$ \\
\hline & & -1 & 8 & 16 & $\sigma_{22}^{2}=1.6400 \ldots$ \\
\hline & $z-(1+i)$ & -1 & 7 & 12 & $1.5823 \ldots$ \\
\hline \multirow[t]{25}{*}{-3} & $z^{2}+j z-1$ & +1 & 06 & 16 & $1.4280 \ldots$ \\
\hline & & +1 & 08 & 12 & $1.5264 \ldots$ \\
\hline & & +1 & 13 & 24 & $1.6750 \ldots$ \\
\hline & & +1 & 15 & 34 & $1.6895 \ldots$ \\
\hline & & -1 & 05 & 12 & $1.6123 \ldots$ \\
\hline & & -1 & 09 & 22 & $1.6595 \ldots$ \\
\hline & & -1 & 14 & 30 & $1.6755 \ldots$ \\
\hline & & $-j$ & 04 & 12 & $1.4986 \ldots$ \\
\hline & & $-j$ & 11 & 26 & $1.6552 \ldots$ \\
\hline & & $+j$ & 08 & 14 & $1.5897 \ldots$ \\
\hline & & $+j$ & 10 & 18 & $1.5674 \ldots$ \\
\hline & & $+\bar{j}$ & 11 & 20 & $1.6219 \ldots$ \\
\hline & & $+\bar{j}$ & 13 & 20 & $1.6710 \ldots$ \\
\hline & & $-\bar{j}$ & 10 & 24 & $1.6604 \ldots$ \\
\hline & & $-\bar{j}$ & 12 & 20 & $1.6336 \ldots$ \\
\hline & $z^{3}-j z^{2}-\bar{j}$ & -1 & 7 & 16 & $1.4672 \ldots$ \\
\hline & & -1 & 8 & 18 & $1.6225 \ldots$ \\
\hline & & $-\bar{j}$ & 4 & 14 & $1.5897 \ldots$ \\
\hline & $z^{3}+\bar{j} z+1$ & +1 & 12 & 20 & $\sigma_{38}^{2}=1.6731 \ldots$ \\
\hline & & -1 & 09 & 20 & $\sigma_{7}^{2}=1.5138$ \\
\hline & & $-j$ & 03 & 12 & $1.6123 \ldots$ \\
\hline & & $-j$ & 10 & 20 & $\sigma_{19}^{2}=1.5907 \ldots$ \\
\hline & & $+\bar{j}$ & 11 & 16 & $\sigma_{22}^{2}=1.6400 \ldots$ \\
\hline & & $-\bar{j}$ & 03 & 12 & 1.6123 \\
\hline & & $-\bar{j}$ & 08 & 20 & $\sigma_{1}^{2}=1.3836$ \\
\hline
\end{tabular}

Remarques. 1. Soit $\tau_{n}$ le nombre de Salem racine du polynôme

$$
A_{n}(z)=z^{n} P_{0}(z) \pm P_{0}^{*}(z)
$$


où $P_{0}(z)=z^{3}-z-1$. Alors $i \tau_{n}$ (resp. $j \tau_{n}$ ) est racine du polynôme

$$
z^{n} P(z) \pm \xi P^{*}(z)
$$

où $\xi=i^{n+3}$ et $P(z)=z^{3}+z+i$ (resp. $\xi=j^{n+3}$ et $P(z)=z^{3}+\bar{j} z+1$ ).

Réciproquement, si $\tau_{n}$ est la racine de valeur absolue $>1$ du polynôme

$$
Q_{n}(z)=z^{n} P(z)+\xi P^{*}(z),
$$

où $\xi$ est une puissance de $i$ (resp. de $j$ ), alors $\tau_{n} / i$ (resp. $\tau_{n} / j$ ) est un nombre de Salem racine de $A_{n}$ si et seulement si $\xi / i^{n+1}\left(\operatorname{resp} . \xi / j^{n}\right)$ est réel.

Ceci explique pourquoi dans le cas où $P(z)=z^{3}+z+i\left(\right.$ resp. $\left.z^{3}+\bar{j} z+1\right)$ on obtient des multiples de nombres de Salem.

Les calculs précédents montrent que la réciproque reste vraie si on suppose $\left|\tau_{n}\right| \leq 1.3$ (resp. $\left|\tau_{n}\right|<\sqrt{1.6123 \ldots}$ ) et sans poser de condition $\operatorname{sur} \xi$.

2. On a la même propriété que précédement dans le cas où $P(z)=$ $z^{4}-i z^{3}-1$ en considérant le polynôme $z^{4}-z^{3}-1$ au lieu du polynôme $P_{0}$.

En conclusion l'application de la construction de Salem aux $\mathbb{Q}(\sqrt{d})$ nombres de Pisot de mesure $<2$ fournit des $\mathbb{Q}(\sqrt{d})$-nombres de Salem non réels de mesure $<1.69$. Les nombres de Salem qu'on peut en déduire (lemme 1) sont parmi ceux qu'on obtient en appliquant la construction de Salem aux nombres de Pisot $<\sqrt{2}$.

\section{Cas cubique totalement réel}

3.1. Soient $K$ un corps cubique totalement réel et $\theta$ un $K$-nombre de Pisot de polynôme minimal $P$ (resp. $F$ ) sur $K$ (resp. sur $\mathbb{Q}$ ). De la définition 2 on déduit que soit $P=F$ et dans ce cas $\pm \theta$ est un nombre de Pisot, soit $P \neq F$ et dans ce cas

$$
F(z)=\prod_{\sigma \in G} \sigma P(z)
$$

Si le polynôme $F$ est réciproque alors il est ou bien de degré 2 et dans ce cas $P=F$, ou bien de degré 6 et dans ce cas $P$ est de degré 2 et $P \neq F$.

Par la suite on suppose que $F$ est non réciproque.

La fraction rationnelle définie par

$$
f(z)=\varepsilon P(z) / P^{*}(z),
$$

où $\varepsilon= \pm 1$ vérifie $f(0)>0$, est une fonction méromorphe sur un ouvert contenant le disque unité, a un seul pôle $1 / \theta$ (qu'on peut supposer positif en changeant au besoin $\theta$ en $-\theta$ ) à l'intérieur du disque unité, admet un développement en série de Taylor au voisinage de l'origine à coefficients entiers de $K$ et est de module 1 sur le cercle unité. 
Désignons par $\left(f_{i}\right)_{i>0}$ la suite des transformées de Schur de $f$ définie par récurrence, à partir de $f_{0}=f$ et aussi longtemps que $\left|f_{i-1}(0)\right|<1$ par la formule

$$
f_{i}(z)=\frac{f_{i-1}(z)-f_{i-1}(0)}{z\left(1-f_{i-1}(z) f_{i-1}(0)\right)} .
$$

Les $f_{i}$ sont alors bornées par 1 sur le cercle unité.

Chamfy [6] a montré qu'il existe un entier $p \geq 0$ tel que $\left|f_{p}(0)\right| \geq 1$. Si $p=0$ alors $f_{0}(0)=f(0) \geq 1$ (ce cas a été traité par Dufresnoy et Pisot [7]) et si $p \geq 1$ alors $0<f(0)<1$ (ce cas a été traité par M. J. Bertin [2]).

Comme dans [2], on note $N_{p}$ l'ensemble des fractions vérifiant les propriétés précédentes.

Rappelons quelques résultats qui nous seront utiles pour déterminer les valeurs possibles de $\theta$ lorsque $P \neq F$ et $M(\theta)<4$.

Lemme 1 [7]. Si $f$ appartient à $N_{0}$ et est de rang $s$ (i.e. $f=\varepsilon P / P^{*}$ et $\operatorname{deg} P=s)$, et si $\sum_{n \geq 0} u_{n} z^{n}$ est son développement en série de Taylor au voisinage de l'origine alors pour $n=1, \ldots, s+1$ (resp. sauf si $n=2$ et $\left.u_{0}=1\right)$ il existe un seul polynôme $D_{n}$ (resp. $\left.D_{n}^{+}\right)$de degré $n$ à coefficients dans $K$ tel que si $E_{n}(z)=-z^{n} D_{n}(1 / z)$ (resp. $\left.E_{n}^{+}(z)=z^{n} D_{n}^{+}(1 / z)\right)$ alors $E_{n}(0)=1$ (resp. $E_{n}^{+}(0)=1$ ) et la fraction $D_{n} / E_{n}\left(\right.$ resp. $\left.D_{n}^{+} / E_{n}^{+}\right)$admet un développement en série de Taylor au voisinage de l'origine de la forme

$$
\begin{gathered}
u_{0}+u_{1} z+\ldots+u_{n-1} z^{n-1}+w_{n} z^{n}+\ldots \\
\text { (resp. } \left.u_{0}+u_{1} z+\ldots+u_{n-1} z^{n-1}+w_{n}^{+} z^{n}+\ldots\right) .
\end{gathered}
$$

En outre, les polynômes $D_{n}$ (resp. $\left.D_{n}^{+}\right)$vérifient les propriétés suivantes:

(1) Pour $n=1, \ldots, s-1$,

$$
D_{n+2}(z)=(1+z) D_{n+1}(z)-z\left(u_{n+1}-w_{n+1}\right) D_{n}(z) /\left(u_{n}-w_{n}\right) .
$$

Pour $n=3, \ldots, s-1$,

$$
D_{n+2}^{+}(z)=(1+z) D_{n+1}^{+}(z)-z\left(u_{n+1}-w_{n+1}^{+}\right) E_{n}^{+}(z) /\left(u_{n}-w_{n}^{+}\right) .
$$

(2) Sauf peut être pour $n=1$ (resp. $n \leq 2)$ les polynômes $D_{n}\left(\right.$ resp. $\left.D_{n}^{+}\right)$ possèdent chacun un zéro unique $\tau_{n}\left(\right.$ resp. $\left.\tau_{n}^{+}\right)$hors du disque unité et les autres à l'intérieur du disque unité et les deux suites $\tau_{n}$ et $\tau_{n}^{+}$vérifient les inégalités

$$
\tau_{1}<\tau_{2}<\ldots<\tau_{s} \leq \tau_{s+1}=\theta=\tau_{s+1}^{+} \leq \tau_{s}^{+}<\tau_{s-1}^{+}<\ldots<\tau_{3}^{+} .
$$

(3) Les quantités $w_{n}$ et $w_{n}^{+}$sont des fonctions rationnelles des $u_{n}$ indépendantes de $f$ vérifiant:

$$
\begin{gathered}
w_{1}<u_{1}, w_{2}<u_{2}, w_{n}<u_{n}<w_{n}^{+} \quad \text { pour } n=3, \ldots, s-1, \\
w_{s}=u_{s}<w_{s}^{+} \text {si } f(1)=1 \quad \text { et } \quad w_{s}<u_{s}=w_{s}^{+} \text {sinon } .
\end{gathered}
$$


(4) Si on note $D_{n}(z)=\sum_{0 \leq i \leq n} a_{i, n} z^{n}$, alors

$$
w_{n}=-1+\sum_{0 \leq i \leq n-1} a_{i, n} u_{i} .
$$

Lemme 2 [2]. Si $f \in N_{p}$ où $p \geq 0$ alors

(1) les polynômes $D_{n}$ (resp. $\left.D_{n}^{+}\right)$définis précédemment existent pour $n=1, \ldots, s+1$ et vérifient les propriétés 1 et 2 du lemme 1 sauf lorsque $f_{p}(0)=-1$ (resp. sauf lorsque $f_{p}(0)=1$ ) et $n=p+2$;

(2) on a les inégalités suivantes:

$$
\left(\theta^{2}+u_{0}\right)\left(u_{0}-1\right) / \theta \leq u_{1} \leq\left(\theta^{2}-u_{0}\right)\left(1+u_{0}\right) / \theta
$$

et pour $n \geq 2$,

$$
w_{n}^{+}-(1+1 / \theta) D_{n}^{+}(\theta)\left(w_{n-1}^{+}-u_{n-1}\right) / D_{n-1}^{+}(\theta) \leq u_{n}
$$

et

$$
u_{n} \leq w_{n}+(1+1 / \theta) D_{n}(\theta)\left(u_{n-1}-w_{n-1}\right) / D_{n-1}(\theta) .
$$

THÉORÈme 4. Soient $K$ un corps cubique totalement réel de discriminant $D_{K}$ et $\pm \theta$ un $K$-nombre de Pisot de mesure $M(\theta)$ et de polynôme minimal $P$ sur $K . S i \pm \theta$ n'est pas un nombre de Pisot alors $M(\theta) \geq 4$ sauf dans les cas suivants :

(a) $D_{K}=81$ et

$$
P(z)=z^{3}-t z^{2}-t \quad \text { ò̀ } \quad t^{3}-3 t^{2}+1=0 \quad \text { et } \quad M(\theta)=3.7508 \ldots
$$

ou bien

$$
P(z)=z^{4}+t z^{3}-1 \quad \text { où } \quad t^{3}-3 t+1=0 \quad \text { et } \quad M(\theta)=3.8035 \ldots ;
$$

(b) $D_{K}=49$ et

$$
P(z)=z^{4}+t z^{3}-1 \quad \text { où } \quad t^{3}-t^{2}-2 t+1=0 \quad \text { et } \quad M(\theta)=3.3555 \ldots
$$

ou bien

$$
P(z)=z^{4}+t z^{3}-1 \quad \text { où } \quad t^{3}-2 t^{2}-t+1=0 \quad \text { et } \quad M(\theta)=3.4931 \ldots
$$

ou bien

$$
P(z)=z^{3}-t z^{2}-t \quad \text { ò̀ } \quad t^{3}-2 t^{2}-t+1=0 \quad \text { et } \quad M(\theta)=3.5041 \ldots
$$

Preuve. Soient donc $K$ un corps cubique totalement réel de discriminant $D_{K}$ et $\theta$ un $K$-nombre de Pisot de mesure $<4$ et qui n'est pas un nombre de Pisot. Du théorème 1 , on déduit $D_{K} \leq 6912$. On connaît d'après [8] tous les corps cubiques de discriminant $\leq 6912$, la détermination est donc ramenée à un nombre fini de corps connus.

Si $P$ est le polynôme minimal de $\theta$ sur $K$ alors $P$ est non réciproque. En effet, si $P$ était réciproque alors $\theta$ serait un entier algébrique totalement réel 
de degré 6 sur $\mathbb{Q}$; un résultat de Schinzel (cf. théorème 2 de [11]) montre dans ce cas que $M(\theta)>((1+\sqrt{5}) / 2)^{3}>4$.

On peut supposer qu'au moins deux conjugués de $\theta$ hors du disque unité sont positifs. En effet, si $\theta$ est un $K$-nombre de Pisot alors $-\theta$ l'est aussi et est de même mesure que $\theta$.

Dans ce qui suit, $\sigma$ et $\tau$ désignent les plongements de $K$ dans $\mathbb{R}$ distincts de l'identité; $\theta, \sigma \theta$ et $\tau \theta$ les conjugués de $\theta$ hors du disque unité et $M$ le produit de ces trois nombres.

Par la suite on distingue deux cas suivant le degré de $P(0)$ sur $\mathbb{Q}$.

1. Cas où $P(0) \in \mathbb{Z}$. Dans ce cas $\theta$ est de degré $\geq 2$ sur $K$. Des inégalités

$$
4>M(\theta) \geq(P(0))^{3},
$$

on déduit $P(0)= \pm 1$.

1.1. Cas où $M>0$. On associe alors à $\theta$ (resp. $\sigma \theta$ et $\tau \theta$ ) la fraction $f(z)=P(0) P(z) / P^{*}(z)$ (resp. $P(0) \sigma P(z) / \sigma P^{*}(z)$ et $P(0) \tau P(z) / \tau P^{*}(z)$ ) qui est un élément de $N_{0}$.

Si $D_{n}$ est un polynôme associé suivant le lemme 1 à la fraction $f$, les deux conjugués de $D_{n}$, à savoir $\sigma D_{n}$ et $\tau D_{n}$ (polynômes dont les coefficients sont les conjuguées par $\sigma$ et $\tau$ des coefficients de $D_{n}$ ), sont aussi associés aux deux fractions conjugués de $f$ suivant le lemme 1 (unicité des polynômes $D_{n}$ ) et les inégalités (2) et (3) du lemme 1 restent vraies par conjugaison, ce qui donne un nombre fini de valeurs pour l'entier $u_{n}$ de $K$. Les entiers algébriques $u_{n}$ de $K$ s'écrivent $u_{n}=i_{n}+j_{n} \alpha+k_{n} \omega$, où $i_{n}, j_{n}$ et $k_{n} \in \mathbb{Z}$ et $\{1, \alpha, \omega\}$ est une base des entiers de $K$. Pour $D_{K} \leq 6912$, la base des entiers est connue [8]. Déterminer $u_{n}$ revient donc à déterminer $i_{n}, j_{n}$ et $k_{n}$.

Si $u_{0}, u_{1}, \ldots, u_{n-1}$ sont connus, les polynômes $D_{1}, \ldots, D_{n}$ le sont aussi d'après le lemme $1(2)$. On calcule $w_{n}$ par le lemme 1(4). Les inégalités

$$
w_{n} \leq u_{n} \leq w_{n}+(1+A)\left(u_{n-1}-w_{n-1}\right) D_{n}(A) /\left(A D_{n-1}(A)\right)
$$

ainsi que les inégalités conjuguées, où $A$ est remplacé respectivement par $B$ et $C$, permettent de déterminer $u_{n}$, puis $D_{n}$ et $w_{n+1}$.

L'inégalité de gauche provient du lemme 1(3); celle de droite résulte de l'inégalité $D_{n+1}(A)<0$, où $A=4, B=2$, et $C=4^{1 / 3}$ sont respectivement des bornes pour $\theta, \sigma \theta$ et $\tau \theta$.

(En fait, on peut choisir des valeurs pour $A, B$ et $C$ qui décroissent avec $n$ comme dans [3], toutefois à cause de la complexité des calculs on préfère les laisser invariants.)

En résolvant successivement les trois premiers systèmes d'inégalités ( $n=1,2$ et 3 ) où $K$ est tel que $D_{K} \leq 6912$, et en ne retenant que les valeurs de $u_{3}$ pour lesquelles le produit des racines $>1$ du polynôme à coefficients rationnels $\left(D_{4}\right)\left(\sigma D_{4}\right)\left(\tau D_{4}\right)$ est $<4$, on trouve $u_{1}=u_{2}=1$ ou bien $u_{1}=1, u_{2}=2$ et $u_{3}=2$ ou 3 . 
- $u_{1}=u_{2}=1$. Ce cas donne des nombres de Pisot [7].

- $u_{1}=1, u_{2}=2$ et $u_{3}=2$. Ce cas donne lieu à un nombre de Pisot. En effet, le polynôme $D_{4}$ s'écrit $D_{4}(z)=(1+z) D_{3}(z)=(1+z)\left(1+z^{2}-z^{3}\right)$; par suite, $\theta$ est racine de $D_{3}$, d'où le résultat.

- $u_{1}=1, u_{2}=2$ et $u_{3}=3$. On détermine de la même manière $u_{4}$ et $u_{5}$, et en éliminant les cas qui donnent des nombres de Pisot on trouve $u_{4}=4$ et $u_{5}=7$, puis $u_{6}=w_{6}=11$, ce qui donne encore un nombre de Pisot. On conclut alors que si les conjugués de $\theta$ hors du disque unité sont de même signe et si $P(0) \in \mathbb{Z}$, alors $M(\theta) \geq 4$.

1.2. Cas où $M<0$. Dans ce cas la fraction $\tau f(-z) \in N_{0}$ et admet comme pôle dans le disque unité $-1 / \tau \theta$. Si $D_{n}$ est associé à la fraction $f$ comme pour le cas précédent alors $\sigma D_{n}$ est associé à $\sigma f$ et $\tau D_{n}(-z)$ est associé à $\tau f(-z)$ seulement lorsque $n$ est pair.

Dans ce cas les inégalités pour $u_{1}, \sigma u_{1}$ et $-\tau u_{1}$ sont identiques au cas précédent, soit :

$0<u_{1}<2(\theta-1 / \theta), \quad 0<\sigma u_{1}<2(\sigma \theta-1 / \sigma \theta), \quad 0<-\tau u_{1}<2((1 / \tau \theta)-\tau \theta)$.

On en déduit que $u_{1}$ engendre $K$ et en résolvant ce système sous la condition du lemme $1(2)$ on trouve $D_{K} \leq 2233$.

On obtient ensuite de manière identique au cas précédent des inégalités pour $u_{2}, \sigma u_{2}$, et $\tau u_{2}$, et en cherchant les $u_{2}$ possibles sous la condition du lemme $1(2)$ on trouve $D_{K} \leq 321$.

On détermine ensuite les $u_{3}$ possibles sous la même condition, on trouve $D_{K} \leq 81$.

Cas $D_{K}=49$. Si $t$ est une racine du polynôme $t^{3}+t^{2}-2 t-1$, alors $\left\{1, t, t^{2}\right\}$ est une base des entiers de $K$. Pour ce cas on a deux valeurs de $u_{1}$ soit $2-t-t^{2}$ et $-t$.

- $u_{1}=2-t-t$. Dans ce cas $u_{2}=5-t-2 t^{2}$ et $u_{3}=13-4 t-6 t^{2}$. En cherchant les valeurs de $u_{4}$ possibles on trouve $u_{4}=w_{4}=30-7 t-13 t^{2}$, ou bien $u_{4}=35-8 t-15 t^{2}$.

Si $u_{4}=w_{4}$ alors $\theta$ est un $K$-nombre de Pisot de degré 4 sur $K$ et de polynôme minimal sur $K,-D_{4}(z)=z^{4}+\left(t^{2}+t-2\right) z^{3}-1$, les conjugués de $\theta$ hors du disque unité sont $1.1736 \ldots, 2.3264 \ldots$ et $-1.2794 \ldots$ et la mesure de $\theta$ est $3.49316 \ldots$

Si $u_{4}=35-8 t-15 t^{2}$, on ne trouve aucune valeur de $u_{5}$ sous la condition du lemme 1(2). On déduit que ce cas ne peut pas avoir lieu.

- $u_{1}=-t$. Dans ce cas $u_{2}=t^{2}$ et $u_{3}=t^{2}-3 t-1$ et on trouve deux valeurs possibles pour $u_{4}$ : ou bien $u_{4}=w_{4}=4 t^{2}-t-1$ ou bien $u_{4}=5 t^{2}-t-1$.

Si $u_{4}=w_{4}$ alors $\theta$ est un $K$-nombre de Pisot ayant pour polynôme minimal sur $K,-D_{4}(z)=z^{4}+t z^{3}-1$. Les conjugués de $\theta$ hors du disque unité sont $1.1328 \ldots, 1.9390 \ldots$ et $-1.5275 \ldots$, et $M(\theta)=3.35556 \ldots$ 
Le cas $u_{4}=5 t^{2}-t-1$ ne donne aucune valeur pour $u_{5}$ sous la même condition que précédemment. On en déduit que ce cas ne peut pas avoir lieu.

Cas $D_{K}=81$. Si $t$ est une racine du polynôme $t^{3}-3 t-1$, alors l'ensemble $\left\{1, t, t^{2}\right\}$ est une base des entiers de $K$.

Pour ce cas on trouve une seule valeur de $u_{1}$, à savoir $u_{1}=-t$, qui correspond à $u_{2}=t^{2}$ et $u_{3}=-4 t-1$. Ce cas donne une seule valeur possible pour $u_{4}, u_{4}=w_{4}=t+4 t^{2}$; et par suite $\theta$ est un $K$-nombre de Pisot ayant pour polynôme minimal sur $K,-D_{4}(z)=z^{4}+t z^{3}-1$. Les conjugués de $\theta$ hors du disque unité sont $1.0995 \ldots, 1.7264 \ldots$ et -2.0036 , et $M(\theta)=3.80357 \ldots$

2. Cas où $P(0) \notin \mathbb{Z}$. Dans ce cas $P(0)$ engendre $K$ et le discriminant du polynôme minimal $Q$ de $P(0)$ sur $\mathbb{Q}$ est $\geq D_{K}$. Des inégalités $4>M(\theta)>$ $M(P(0))$, on déduit que les coefficients de $Q$ sont bornés et que $P(0)$ ne prend qu'un nombre fini de valeurs.

En utilisant les inégalités de Mahler liant les coefficients et la mesure de $Q$ et en ne gardant que les polynômes irréductibles à racines réelles tels que $Q(0)>0$ on obtient 21 valeurs possibles pour $Q$.

Pour chacune de ces valeurs on donne (par ordre croissant de mesure) sur la même ligne du tableau 2 et dans l'ordre suivant les coefficients du polynôme $Q$, son discriminant et une valeur approchée de ses racines.

Tableau 2

\begin{tabular}{llllllll}
\hline 1 & -2 & -1 & 1 & 049 & -0.80 & 0.55 & 2.24 \\
1 & -1 & -2 & 1 & 049 & -1.24 & 0.44 & 1.80 \\
1 & -3 & +0 & 1 & 081 & -0.53 & 0.65 & 2.87 \\
1 & +0 & -3 & 1 & 081 & -1.87 & 0.34 & 1.53 \\
1 & -2 & -2 & 2 & 148 & -1.17 & 0.68 & 2.48 \\
1 & -3 & -1 & 2 & 229 & -0.86 & 0.74 & 3.11 \\
1 & -3 & -1 & 1 & 148 & -0.67 & 0.46 & 3.21 \\
1 & -1 & -3 & 1 & 148 & -1.48 & 0.31 & 2.17 \\
1 & -3 & +0 & 3 & 081 & -0.87 & 1.34 & 2.53 \\
1 & -3 & -2 & 1 & 257 & -0.83 & 0.34 & 3.49 \\
1 & -2 & -3 & 1 & 257 & -1.19 & 0.28 & 2.91 \\
1 & -4 & +1 & 1 & 169 & -0.37 & 0.72 & 3.65 \\
1 & +1 & -4 & 1 & 169 & -2.65 & 0.27 & 1.37 \\
1 & +0 & -4 & 2 & 148 & -2.21 & 0.53 & 1.67 \\
1 & -3 & -2 & 3 & 473 & -1.28 & 0.79 & 3.33 \\
1 & -2 & -3 & 2 & 316 & -1.34 & 0.52 & 2.81 \\
1 & -4 & +0 & 2 & 404 & -0.65 & 0.78 & 3.86 \\
1 & -4 & +0 & 1 & 229 & -0.47 & 0.53 & 3.93 \\
1 & +0 & -4 & 1 & 229 & -2.11 & 0.25 & 1.86 \\
1 & -2 & -3 & 3 & 321 & -1.46 & 0.76 & 2.69 \\
1 & -3 & -1 & 4 & 229 & -1.11 & 1.25 & 2.86 \\
\hline
\end{tabular}


On en déduit que $D_{K} \leq 473$, que si $K=\mathbb{Q}(P(0))$ alors le discriminant du polynôme minimal de $P(0)$ sur $\mathbb{Q}$ est égal à $D_{K}$ (on déduit alors que la partie $\left\{1, P(0), P(0)^{2}\right\}$ est une base des entiers de $K$ ) et que l'entier de $K$, $\varepsilon P(0)$ où $\varepsilon= \pm 1$, ne peut être un $K$-nombre de Pisot de mesure $<4$ (si $M(P(0))=4$ alors $P(0)$ ne peut être un $\mathbb{Q}(P(0))$-nombre de Pisot sauf s'il est racine du polynôme $z^{3}-3 z^{2}-z+4$ qui est de discriminant 229$)$; et par suite $P(0)$ admet au moins un conjugué de module $<1$.

Un simple calcul montre que les entiers cubiques totalement positifs sont de mesure $\geq M(t)>5.0489 \ldots$, où $t$ est racine du polynôme $t^{3}-5 t^{2}+6 t+1$; on en déduit que les conjugués de $P(0)$ ne sont pas de même signe car sinon

$$
M(P(0))=M(|P(0)|)>5 .
$$

D'autre part, $P(0)$ admet au moins un conjugué (on le note encore $P(0)$ ) en valeur absolue $>1$; si $u_{0}=\varepsilon P(0)>1$ où $\varepsilon= \pm 1$, alors les conjugués de $u_{0}, \sigma u_{0}$ et $\tau u_{0}$ où $\sigma$ et $\tau$ désignent les deux plongements de $K$ dans $\mathbb{R}$ distincts de l'identité, ne peuvent pas être tous les deux dans l'intervalle ] $-1,0\left[\right.$. Sinon l'entier totalement positif $u_{0}+1$ serait un nombre de Pisot et dans ce cas

$$
M\left(u_{0}+1\right)=u_{0}+1>5,
$$

et par suite $M\left(u_{0}\right)=u_{0}>4$. On distingue alors 3 cas possibles suivant les valeurs de $\sigma u_{0}$ et $\tau u_{0}$.

2.1. Cas où $u_{0}$ admet au moins deux conjugués supérieurs à 1 . Si on note $\tau u_{0}$ le conjugué de $u_{0}$ supérieur à 1 , le tableau 2 montre que ce cas n'a lieu que si $D_{K}=81$ et avec ces notations on a

$$
u_{0}=2.53 \ldots, \quad \sigma u_{0}=-0.87 \ldots \quad \text { et } \tau u_{0}=1.34 \ldots
$$

2.1.1. Cas où $M>0$. Si on associe à $\theta$ (resp. $\sigma \theta$ et $\tau \theta$ ) la fraction $f$ (resp. $\sigma f$ et $\tau f$ ) comme pour le cas où $P(0) \in \mathbb{Z}$, alors les deux fractions $f$ et $\tau f \in N_{0}$ et il existe un entier $p \geq 1$ tel que la fraction $-\sigma f \in N_{p}$. (Par la suite on dira simplement que $f \in N_{p}$ où $p$ désigne un entier naturel non nul.)

Des inégalités

$$
\theta>u_{0}, \quad \sigma \theta>1, \quad \tau \theta>\tau u_{0} \quad \text { et } \quad M(\theta)<4,
$$

on déduit

$$
\theta<2.97, \quad \sigma \theta<1.18 \text { et } \tau \theta<1.58 \text {. }
$$

Le lemme 1(2),(3) ainsi que le lemme 2(2) entraînent alors les inégalités suivantes pour $u_{1}, \sigma u_{1}$ et $\tau u_{1}$ :

$$
5.4<u_{1}<7.4, \quad-0.82<\sigma u_{1}<0.24 \text { et } 0.8<\tau u_{1}<1.71 .
$$

En résolvant ce système on trouve que $u_{1}$ ne peut prendre qu'une seule valeur, soit $u_{1}=w_{1}$, et ce cas n'est pas possible puisque $\theta$ est de degré $\geq 2$ 
(en fait, $\theta$ est de degré $\geq 3$ car si $\theta$ était de degré 2 alors il serait totalement réel et la conclusion serait la même que pour le cas où $P$ est réciproque).

2.1.2. Cas où $M<0$. Ici trois cas se présentent: le cas où $\theta<0$, le cas où $\sigma \theta<0$ et le cas où $\tau \theta<0$.

Si $\theta<0$ ou bien $\tau \theta<0$ alors on considère respectivement les fractions $f(-z)$ ou $\tau f(-z)$ qui sont des éléments de $N_{0}$ et si $\sigma \theta<0$ on considère la fraction $-\sigma f(-z)$ qui est un élément de $N_{p}$, et les mêmes calculs que précédemment montrent que ce cas ne peut pas avoir lieu.

2.2. Cas où $u_{0}$ admet un conjugué inférieur à -1 noté $\tau u_{0}$ et un conjugué positif noté $\sigma u_{0}$. Le tableau 2 montre que ce cas n'a lieu que pour les corps de discriminant $49,81,169,229,257,316,321$ et 473 avec une seule valeur pour $u_{0}$ et dans le corps de discriminant 148 avec 3 valeurs pour $u_{0}$.

Comme la partie $\left\{1, u_{0}, u_{0}^{2}\right\}$ est une base des entiers du corps $K=\mathbb{Q}\left(u_{0}\right)$, on exprimera pour ce cas ainsi que pour les cas qui vont suivre les $\left(u_{k}\right)_{k \geq 0}$ dans cette base, chose qui permet de simplifier les calculs.

2.2.1. Cas où $M>0$. Dans ce cas les fractions $f$ et $-\tau f$ sont des éléments de $N_{0}$ et la fraction $\sigma f$ est un élément de $N_{p}$. Du lemme 1(2), (3) ainsi que du lemme 2(2), on déduit que seuls les $u_{0}$ de discriminant 49 et 148 donnent des valeurs possibles pour $u_{1}$ à savoir :

Si $D_{K}=49$,

$$
\begin{array}{lll}
u_{0}=1.80 \ldots, & \sigma u_{0}=0.44 \ldots, & \tau u_{0}=-1.24 \ldots, \\
u_{1}=4.04 \ldots, & \sigma u_{1}=-0.35 \ldots, & \tau u_{1}=-0.69 \ldots
\end{array}
$$

Si $D_{K}=148$,

$$
\begin{array}{lll}
u_{0}=2.48 \ldots, & \sigma u_{0}=0.68 \ldots, & \tau u_{0}=-1.17 \ldots, \\
u_{1}=7.63 \ldots, & \sigma u_{1}=0.16 \ldots, & \tau u_{1}=-0.80 \ldots
\end{array}
$$

Du lemme 1(2), (3) ainsi que du lemme 2(2), on déduit que le deuxième cas donne une seule valeur possible pour $u_{2}$, soit $u_{2}=w_{2}$, cas qui ne peut pas avoir lieu car $\theta$ est de degré $\geq 3$.

Quant au premier cas, il donne $u_{2}=w_{2}$ ou bien $u_{2}=3 u_{0}^{2}+3 u_{0}-2$. La première valeur de $u_{2}$ n'est pas possible et la seconde ne donne aucune valeur pour $u_{3}$.

2.2.2. Cas où $M<0$. Ici trois cas sont à envisager :

- $\theta<0$. Dans ce cas la fraction $f(-z)$ est un élément de $N_{0}$. Le raisonnement est le même que pour le cas précédent. On trouve que seuls les $u_{0}$ de discriminant 49, 81, et 148 donnent des valeurs possibles pour $u_{1}$, et que seul le $u_{0}$ de discriminant 49 donne une valeur pour $u_{2}$, à savoir : 


$$
\begin{array}{lll}
u_{0}=1.80 \ldots, & \sigma u_{0}=0.44 \ldots, & \tau u_{0}=-1.24 \ldots, \\
u_{1}=-3.24 \ldots, & \sigma u_{1}=-0.19 \ldots, & \tau u_{1}=-1.55 \ldots, \\
u_{2}=7.65 \ldots, & \sigma u_{2}=0.53 \ldots, & \tau u_{2}=-3.18 \ldots
\end{array}
$$

De la même manière que pour le cas précédent, ce cas ne donne aucune valeur possible pour $u_{3}$.

- $\sigma \theta<0$. Dans ce cas la fraction $\sigma f(-z)$ est un élément de $N_{p}$. Seuls les $u_{0}$ de discriminant 49 et 148 donnent des valeurs possibles pour $u_{1}$ et seul le $u_{0}$ de discriminant 49 donne une valeur de $u_{2}$ et les valeurs de $u_{0}, u_{1}, u_{2}$ ainsi que celles de leurs conjugués sont identiques au cas où $\theta>0, \sigma \theta>0$, $\tau \theta>0$. Ce cas aussi ne donne aucune valeur possible pour $u_{3}$.

- $\tau \theta<0$. Dans ce cas la fraction $-\tau f(-z)$ est un élément de $N_{0}$. Seul les $u_{0}$ de discriminant 49,81 et 148 donnent des valeurs possibles pour $u_{1}$ et seul le $u_{0}$ de discrimiant 49 donne une valeur pour $u_{2}$. Les valeurs de $u_{0}$, $-u_{1}$ et $u_{2}$ sont identiques respectivement aux valeurs de $u_{0}, u_{1}$ et $u_{2}$ du cas $\theta<0$. Ce cas donne une valeur pour $u_{3}$, soit $u_{3}=17.03 \ldots, \sigma u_{3}=0.43 \ldots$, $\tau u_{3}=5.52 \ldots$

La racine $>1 \mathrm{du}$ polynôme $D_{4}\left(\right.$ resp. $\left.\sigma D_{4}, d_{4}\right)$ où $D_{4}\left(\right.$ resp. $\left.\sigma D_{4}, d_{4}\right)$ est associé suivant le lemme 1 à la fraction $f$ (resp. $\sigma f,-\tau f(-z)$ ), vaut $2.21 \ldots$ (resp. $1.03 \ldots, 1.73 \ldots$ ); et ce cas ne donne aucune valeur de $u_{4}$.

On conclut pour ce cas que $M(\theta) \geq 4$.

2.3. Cas où $u_{0}$ admet un conjugué compris entre 0 et 1 noté $\sigma u_{0}$ et un conjugué compris entre -1 et 0 noté $\tau u_{0}$. Le tableau 2 montre que ce cas a lieu pour les corps de discriminant 49, 81, 148, 169, 257 et 404 avec une seule valeur de $u_{0}$ et le corps de discriminant 229 avec deux valeurs de $u_{0}$.

2.3.1. Cas où $M>0$. Dans ce cas la fraction $f$ est un élément de $N_{0}$ et les fractions $\sigma f$ et $-\tau f$ sont des éléments de $N_{p}$.

Du lemme 1(2), (3) ainsi que du lemme 2(2), on déduit que seuls les $u_{0}$ de discriminant 49, 81, 148 et 169 donnent des valeurs possibles pour $u_{1}$ et que toutes ces valeurs de $u_{0}$ ne donnent aucune valeur possible pour $u_{2}$.

Supposons maintenant que les conjugués de $\theta$ hors du disque unité ne soient pas de même signe.

2.3.2. Cas où $M<0$. Ici trois cas sont à envisager :

- $\theta<0$. Dans ce cas la fraction $f(-z)$ est un élément de $N_{0}$. Le raisonnement est le même que précédemment, on trouve que seuls les $u_{0}$ de discriminant 49, 81 et 148 donnent des valeurs pour $u_{1}$ et que seul le $u_{0}$ de discriminant 49 donne des valeurs pour $u_{2}$, à savoir : 


$$
\begin{array}{rcll} 
& u_{0}=2.24 \ldots, & \sigma u_{0}=0.55 \ldots, & \tau u_{0}=-0.80 \ldots, \\
\text { et } & \left(u_{1}=-9.09 \ldots,\right. & \sigma u_{1}=0.38 \ldots, & \tau u_{1}=-0.28 \ldots \\
\text { et } & u_{2}=31.78 \ldots, & \sigma u_{2}=-0.04 \ldots, & \tau u_{2}=-0.74 \ldots \\
\text { ou bien } & u_{2}=36.83 \ldots, & \sigma u_{2}=0.26 \ldots, & \left.\tau u_{2}=-0.10 \ldots\right) \\
\text { ou bien } & \left(u_{1}=-5.04 \ldots,\right. & \sigma u_{1}=-0.30 \ldots, & \tau u_{1}=-0.64 \ldots \\
\text { et } & u_{2}=15.39 \ldots, & \sigma u_{2}=-0.52 \ldots, & \left.\tau u_{2}=-0.87 \ldots\right) .
\end{array}
$$

Du lemme 1(2), (3) ainsi que du lemme 2(2) on déduit qu'aucun de ces cas ne peut donner une valeur de $u_{3}$ possible.

- $\sigma \theta<0$. Dans ce cas la fraction $\sigma f(-z)$ est un élément de $N_{p}$. Le raisonnement est le même que précédemment; on trouve que seuls les $u_{0}$ de discriminant 49, 81 et 148 donnent des valeurs pour $u_{1}$ et qu'aucun d'eux ne donnent de valeur pour $u_{2}$.

- $\tau \theta<0$. Dans ce cas la fraction $-\tau f(-z)$ est un élément de $N_{p}$. Le raisonnement est le même que précédemment; on trouve que seuls les $u_{0}$ de discriminant 49, 81, 148, 169 et 229 donnent des valeurs possibles pour $u_{1}$ et que seuls les $u_{0}$ de discriminant 49 et 81 donnent des valeurs pour $u_{2}$.

Cas $D_{K}=81$ :

$$
\begin{array}{lll}
u_{0}=2.87 \ldots, & \sigma u_{0}=0.65 \ldots, & \tau u_{0}=-0.53 \ldots, \\
u_{1}=8.29 \ldots, & \sigma u_{1}=0.42 \ldots, & \tau u_{1}=0.28 \ldots, \\
u_{2}=26.75 \ldots, & \sigma u_{2}=0.93 \ldots, & \tau u_{2}=-0.68 \ldots
\end{array}
$$

En cherchant les valeurs possibles pour $u_{3}$ on trouve $u_{3}=w_{3}=84.32 \ldots$ On déduit alors que $\theta$ est $K$-nombre de Pisot de polynôme minimal sur $K$

$$
-D_{3}(z)=z^{3}-t z^{2}-t \quad \text { où } t^{3}-3 t^{2}+1=0,
$$

les conjugués de $\theta$ hors du disque unité sont $3.1665 \ldots, 1.1479 \ldots$ et $-1.0318 \ldots$, et $M(\theta)=3.7508 \ldots$

Cas $D_{K}=49$. Les quantités $u_{0},-u_{1}$ et $u_{2}$ prennent respectivement les valeurs que prennent les $u_{0}, u_{1}$ et $u_{2}$ du cas $\theta<0$ et de plus une autre valeur pour $u_{2}$ lorsque $u_{1}=5.04 \ldots$, soit $u_{2}=13.59 \ldots, \sigma u_{2}=0.72 \ldots$ et $\tau u_{2}=-1.31 \ldots$

De la même manière que pour les cas précédents ces cas ne donnent aucune valeur de $u_{3}$ et $u_{4}$ sauf le dernier cas qui donne $u_{3}=w_{3}=34.58 \ldots$ On déduit alors que $\theta$ est un $K$-nombre de Pisot de polynôme minimal sur $K$

$$
-D_{3}(z)=z^{3}-t z^{2}-t \quad \text { où } t^{3}-2 t^{2}-t+1=0,
$$

les conjugués de $\theta$ hors du disque unité sont $2.5836 \ldots, 1.0542 \ldots$ et $-1.2864 \ldots$, et $M(\theta)=3.5041 \ldots$

Ceci achève la preuve du théorème. 
3.2. Application à la détermination de polynômes réciproques de petite mesure. En appliquant la construction de Salem aux $K$-nombres de Pisot de mesure $<4$ cités dans l'énoncé du théorème précédent, on obtient des polynômes réciproques ayant $j$ racines hors du disque unité, où $1 \leq j \leq 3$.

Si on note $P$ le polynôme minimal sur $K$ d'un tel $K$-nombre de Pisot, on regarde la valeur absolue $M_{n}$ du produit des racines hors du disque unité du polynôme $R_{n}$ défini par

$$
R_{n}(z)=\prod_{\sigma \in G}\left(z^{n} \sigma P(z)+\xi \sigma P^{*}(z)\right),
$$

où $\xi= \pm 1$ et $n \in \mathbb{N}^{*}$.

Pour les calculs on s'est limité aux cas où $M_{n} \leq 3$ et le lemme suivant montre que la valeur 16 est une borne supérieure de $n$ suffisante.

Lemme 3. Avec les notations précédentes et si $n>16$ alors $M_{n}>3$.

Preuve. Avec les mêmes notations et d'après le théorème 4, le nombre $\theta$ ne prend qu'un nombre fini de valeurs.

Si $\alpha$ est un conjugué de $\theta$ sur $K$ de module $<1$, alors de l'égalité

$$
|z-\alpha|^{2}-|1-\alpha z|^{2}=\left(|z|^{2}-1\right)\left(1-|\alpha|^{2}\right)
$$

on déduit pour $|z|=\varrho>1$ et lorsque le cercle $|z|=\varrho$ ne contient aucun des inverses des conjugués de $\theta$ l'inégalité

$$
\left|P(z) / P^{*}(z)\right|>|(z-\theta) /(1-\theta z)| .
$$

De la preuve de la construction de Salem [4] on a pour $|z|=\varrho<|\theta|$ l'inégalité

$$
|(z-\theta) /(1-\theta z)|>(|\theta|-\varrho) /(\varrho|\theta|-1),
$$

d'où l'inégalité pour $|z|=\varrho$

$$
\left|z^{n} P(z) / P^{*}(z)\right|>\varrho^{n}(|\theta|-\varrho) /(\varrho|\theta|-1)=m_{n}(\theta) .
$$

Le calcul qui va suivre montre qu'on peut choisir une valeur minimale $>1$ pour $m_{n}(\theta)$ lorsque $n>16$.

Ensuite du théorème de Rouché on déduit que le polynôme

$$
T_{n}(z)=z^{n} P(z)+\xi P^{*}(z)
$$

admet une racine de valeur absolue supérieure à $\varrho$ lorsque $n>16$.

Si l'on considère le conjugué de $\theta$ hors du disque unité $\sigma \theta$ (resp. $\tau \theta$ ) et si on lui associe la quantité $\sigma \varrho$ (resp. $\tau \varrho$ ) de la même manière qu'on associe $\varrho$ à $\theta$, alors le polynôme conjugué $\sigma T_{n}$ (resp. $\tau T_{n}$ ) de $T_{n}$ admet une racine de valeur absolue $>\sigma \varrho($ resp. $\tau \varrho)$.

Il suffit maintenant de choisir $\varrho, \sigma \varrho$ et $\tau \varrho$ tels que leur produit soit $>3$, pour déduire le résultat.

Le calcul. On vérifie d'abord que les valeurs absolues des conjugués de $\theta$ sur $\mathbb{Q}$ ne sont pas des rationnels et on choisit alors $\varrho$ dans $\mathbb{Q}$. 
- $M(\theta)=3.75 \ldots$ L'un des conjugués de $\theta$ (qu'on note $\theta$ ) a pour valeur absolue $3.1665 \ldots$ Si on choisit $\varrho=3.1$ alors pour $n \geq 5$, on a $m_{n}(\theta)>2.147$ et $M_{n}>3.1$.

- $M(\theta)=3.50 \ldots$ Deux conjugués de $\theta$ (qu'on note $\theta$ et $\sigma \theta$ ) ont pour valeurs absolues $2.5836 \ldots$ et $1.2864 \ldots \mathrm{Si}$ on choisit $\varrho=2.51$ et $\sigma \varrho=1.2$ alors on obtient $m_{n}(\theta)>1.336$ lorsque $n \geq 5$ et $m_{n}(\sigma \theta)>1.1804$ lorsque $n \geq 11$, et par suite $M_{n}>3.01$ lorsque $n \geq 11$.

- $M(\theta)=3.80 \ldots$ De manière identique au cas précédent et en choisissant $\varrho=2$ et $\sigma \varrho=1.7$ on obtient pour $n \geq 10$, l'inégalité $M_{n}>3.4$.

- $M(\theta)=3.49 \ldots$ Les trois conjugués de $\theta$ qu'on note $\theta, \sigma \theta$ et $\tau \theta$ ont pour valeurs absolues respectivement $2.3264 \ldots, 1.2794 \ldots$ et $1.1736 \ldots \mathrm{Si}$ on choisit $\varrho=2.3, \sigma \varrho=1.2$ et $\tau \varrho=1.1$ on trouve $m_{n}(\theta)>2.06$ si $n \geq 7$, $m_{n}(\sigma \theta)>1.1$ si $n \geq 11$ et $m_{n}(\tau \theta)>1.05$ si $n \geq 15$, et par suite $M_{n}>3.036$ lorsque $n \geq 15$.

- $M(\theta)=3.35 \ldots$ De manière identique au cas précédent et en choisissant $\varrho=1.93, \sigma \varrho=1.03$ et $\tau \varrho=1.52$ on trouve $M_{n}>3.02$ pour $n>16$ et ceci achève la preuve du lemme.

Par la suite on donne tous les polynômes réciproques irréductibles non cyclotomiques de mesure $\leq 3$ obtenus en affectant à $\theta$ les valeurs de l'énoncé du théorème 4, à $n$ les entiers de 1 à 16 (en fait, pour chaque cas on choisit pour $n$ la borne correspondante trouvée dans la preuve du lemme précédent au lieu de 16) et à $\xi$ les valeurs \pm 1 .

Pour chaque polynôme $P$, on précise dans l'ordre suivant et sur la même ligne du tableau 3 l'entier $\xi$, l'entier $n$, le degré du polynôme réciproque obtenu et enfin ses racines hors du disque unité.

Tableau 3

\begin{tabular}{|c|c|c|c|c|c|c|}
\hline$\underline{D_{K}}$ & $P(z)$ & & & & & \\
\hline 81 & 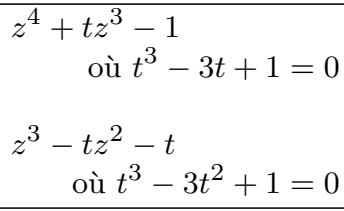 & $\begin{array}{l}+1 \\
+1 \\
-1 \\
-1 \\
-1\end{array}$ & $\begin{array}{l}1 \\
3 \\
4 \\
2 \\
3\end{array}$ & $\begin{array}{l}12 \\
18 \\
18 \\
12 \\
12\end{array}$ & $\begin{array}{l}2.9758 \ldots \\
2.0225 \ldots \\
1.8455 \ldots \\
1.7358 \ldots \\
2.8406 \ldots\end{array}$ & $\begin{array}{l} \\
-1.4371 \ldots \\
-1.1738 \ldots\end{array}$ \\
\hline 49 & $\begin{array}{l}z^{4}+t z^{3}-1 \\
\text { où } t^{3}+t^{2}-2 t-1=0\end{array}$ & & $\begin{array}{l}01 \\
03 \\
09 \\
11 \\
13 \\
15 \\
03\end{array}$ & $\begin{array}{l}06 \\
18 \\
36 \\
42 \\
24 \\
36 \\
18\end{array}$ & $\begin{array}{l}+2.3821 \ldots \\
+1.7790 \ldots \\
+1.5502 \ldots \\
+1.5377 \ldots \\
+1.5320 \ldots \\
+1.5295 \ldots \\
-1.3433 \ldots\end{array}$ & $\begin{array}{l} \\
-1.5076 \ldots \\
-1.9341 \ldots \\
-1.9377 \ldots \\
-1.9387 \ldots \\
-1.93899 \ldots \\
-2.1485 \ldots\end{array}$ \\
\hline
\end{tabular}


Tableau 3 (suite)

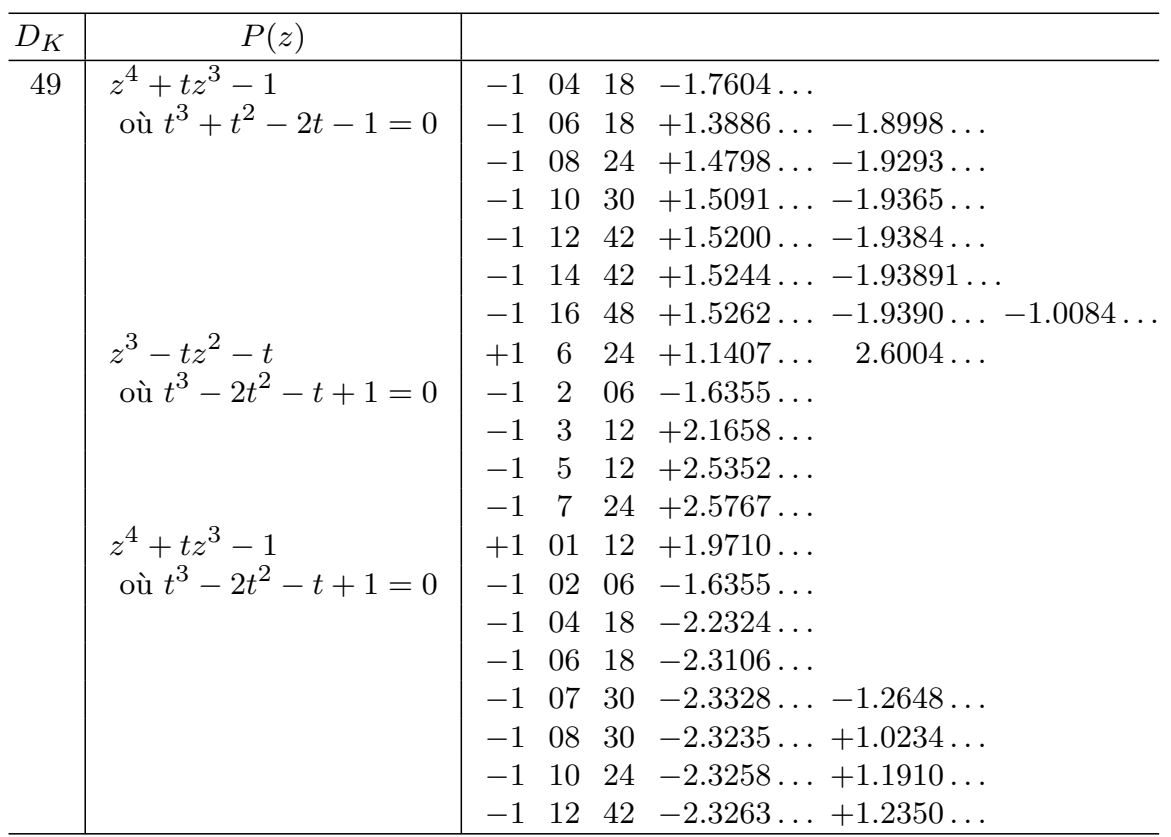

R e mar que. L'application de la construction de Salem aux $K$-nombres de Pisot énoncés dans le théorème 4 permet d'obtenir des polynômes réciproques de petite mesure.

Ces mesures semblent augmenter avec le discriminant du corps $K$. Par exemple le plus petit nombre de Salem qu'on obtient par cette méthode au dessus du corps de discriminant 49 est $1.6355 \ldots$ alors que le plus petit nombre de Salem qu'on obtient au dessus du corps de discriminant 81 est $2.0225 \ldots$

De même, on obtient un $K$-nombre de Salem de mesure $<3$ au dessus du corps de discriminant 49, ce qui n'est pas le cas pour le corps de discriminant 81. Un analogue du théorème 1 pour des polynômes réciproques pourrait expliquer ceci.

\section{Bibliographie}

[1] A. M. Bergé et J. Martinet, Notions relatives de régulateurs et de hauteurs, Acta Arith. 54 (1989), 155-170.

[2] M. J. Bertin, K-nombres de Pisot et de Salem, ibid. 68 (1994), 113-131.

[3] - K-nombres de Pisot et de Salem, dans : Advances in Number Theory, F. Q. Gouvêa et N. Yui (eds.), Proceedings of the third Conference of the Canadian Number Theory Association, Oxford University Press, 1993, 391-397. 
[4] M. J. Bertin et M. Pathiaux-Delefosse, Conjecture de Lehmer et petits nombres de Salem, Queen's Papers in Pure and Appl. Math. 81, Kingston, 1989.

[5] D. W. Boyd, Small Salem numbers, Duke Math. J. 44 (1977), 315-327.

[6] C. Chamfy, Fonctions méromorphes dans le cercle unité et leurs séries de Taylor, Ann. Inst. Fourier (Grenoble) 8 (1958), 211-261.

[7] M. M. J. Dufresnoy et C. Pisot, Etude de certaines fonctions méromorphes bornées sur le cércle unité, Ann. Sci. École Norm. Sup. 70 (1955), 69-92.

[8] M. Olivier, Tables de corps cubiques réels de discriminant inférieur à 2000000, ftp anonyme à l'URL "ftp://megrez.math.u-bordeaux.fr/pub/numberfields".

[9] Pari Calculator, par C. Batut, D. Bernardi, H. Cohen et M. Olivier, copyright 1989, 1992.

[10] C. Pisot, Quelques aspects de la théorie des entiers algébriques, Presses de l'Université de Montréal, 1963.

[11] A. Schinzel, On the product of the conjugates outside the unit circle of an algebraic number, Acta Arith. 24 (1973), 385-399.

Department of Mathematics

College of Sciences

King Saud University

P.O. Box 2455

Riyadh 1145, Saudi Arabia

E-mail: f40m009@saksu.bitnet

Reçu le 12.1.1995

et révisé le 2.11.1995 